\title{
Excessive Laughter-like Vocalizations, Microcephaly, and Translational Outcomes in the Ube3a Deletion Rat Model of Angelman Syndrome
}

\author{
${ }^{\text {DE}}$ Elizabeth L. Berg, ${ }^{1}$ Shekib A. Jami, ${ }^{2}$ Stela P. Petkova, ${ }^{1}$ Annuska Berz,${ }^{3,4}$ Timothy A. Fenton, ${ }^{1}$ \\ Jason P. Lerch, ${ }^{5,6,7}$ David J. Segal, ${ }^{8}{ }^{\circledR}$ John A. Gray, ${ }^{2}{ }^{\circledR}$ Jacob Ellegood, ${ }^{5}{ }^{\circledR}$ Markus Wöhr, $, 3,4,9,10$ and \\ Jill L. Silverman ${ }^{1}$ \\ ${ }^{1}$ MIND Institute and Department of Psychiatry and Behavioral Sciences, University of California Davis School of Medicine, Sacramento, California 95817, \\ ${ }^{2}$ Department of Neurology, Center for Neuroscience, University of California Davis, Davis, California 95618, ${ }^{3}$ Behavioral Neuroscience, Experimental and \\ Biological Psychology, Faculty of Psychology, Philipps-University Marburg, Marburg, 35032, Germany, ${ }^{4}$ Center for Mind, Brain and Behavior, Philipps- \\ University Marburg, Marburg, 35032, Germany, ${ }^{5}$ Mouse Imaging Centre, Hospital for Sick Children, Toronto, Ontario M5T 3H7, Canada, ${ }^{6}$ Department of \\ Medical Biophysics, University of Toronto, Toronto, Ontario M5S, Canada, ${ }^{7}$ Wellcome Centre for Integrative Neuroimaging, University of Oxford, Oxford, \\ OX3 9DU, United Kingdom, ${ }^{8} \mathrm{MIND}$ Institute, Genome Center, and Department of Biochemistry and Molecular Medicine, University of California Davis, \\ Davis, California 95616, ${ }^{9} \mathrm{KU}$ Leuven, Faculty of Psychology and Educational Sciences, Research Unit Brain and Cognition, Laboratory of Biological \\ Psychology, Social and Affective Neuroscience Research Group, Leuven, B-3000, Belgium, and ${ }^{10} \mathrm{KU}$ Leuven, Leuven Brain Institute, Leuven, B-3000, Belgium
}

Angelman syndrome (AS) is a rare genetic neurodevelopmental disorder characterized by intellectual disabilities, motor and balance deficits, impaired communication, and a happy, excitable demeanor with frequent laughter. We sought to elucidate a preclinical outcome measure in male and female rats that addressed communication abnormalities of AS and other neurodevelopmental disorders in which communication is atypical and/or lack of speech is a core feature. We discovered, and herein report for the first time, excessive laughter-like $50 \mathrm{kHz}$ ultrasonic emissions in the Ube $3 a^{\text {mat-/pat }+}$ rat model of AS, which suggests an excitable, playful demeanor and elevated positive affect, similar to the demeanor of individuals with AS. Also in line with the AS phenotype, Ube $3 a^{\text {mat }- \text { pat }+}$ rats $^{\text {ats }}$ demonstrated aberrant social interactions with a novel partner, distinctive gait abnormalities, impaired cognition, an underlying LTP deficit, and profound reductions in brain volume. These unique, robust phenotypes provide advantages compared with currently available mouse models and will be highly valuable as outcome measures in the evaluation of therapies for AS.

Key words: Angelman syndrome; behavior; play; rat; Ube3a; ultrasonic vocalization

Significance Statement

Angelman syndrome (AS) is a severe neurogenetic disorder for which there is no cure, despite decades of research using mouse models. This study used a recently developed rat model of AS to delineate disease-relevant outcome measures to facilitate therapeutic development. We found the rat to be a strong model of AS, offering several advantages over mouse models by exhibiting numerous AS-relevant phenotypes, including overabundant laughter-like vocalizations, reduced hippocampal LTP, and volumetric anomalies across the brain. These findings are unconfounded by detrimental motor abilities and background strain, issues plaguing mouse models. This rat model represents an important advancement in the field of AS, and the outcome metrics reported herein will be central to the therapeutic pipeline.

Received Apr. 29, 2021; revised Aug. 23, 2021; accepted Aug. 26, 2021.

Author contributions: E.L.B., S.A.J., S.P.P., J.P.L., D.J.S., J.A.G., J.E., M.W., and J.L.S. designed research; E.L.B., S.A.J., S.P.P., A.B., T.A.F., J.E., and M.W. performed research; E.L.B., S.A.J., S.P.P., A.B., J.P.L., J.A.G., J.E., M.W., and J.L.S. analyzed data; E.L.B., S.A.J., S.P.P., J.E., M.W., and J.L.S. wrote the first draft of the paper; E.L.B., S.A.J., S.P.P., A.B., T.A.F., J.P.L., D.J.S., J.A.G., J.E., M.W., and J.L.S. edited the paper; E.L.B. wrote the paper.

This work was supported by the Foundation for Angelman Syndrome Therapeutics to E.L.B., D.J.S., and J.LS.; the MIND Institute's Intellectual and Developmental Disabilities Resource Center National Institutes of Health U54HD079125 to J.L.S.; National Institute of Mental Health R01MH117130 to J.A.G.; Deutsche Forschungsgemeinschaft W0 1732/4-2 to M.W.; Canadian Institute for Health Research to J.P.L. and J.E.;
Ontario Brain Institute to J.P.L. and J.E.; and Gustav Adolf Lienert Foundation to A.B. We thank Matthew Matson, Ruona Lin, Steven Xiong, Yutian Shen, and Anurupa Kar for technical support.

The authors declare no competing financial interests.

Correspondence should be addressed to Jill L. Silverman at jsilverman@ucdavis.edu.

https://doi.org/10.1523/NNEUROSC1.0925-21.2021

Copyright $\odot 2021$ Berg et al.

This is an open-access article distributed under the terms of the Creative Commons Attribution 4.0 International license, which permits unrestricted use, distribution and reproduction in any medium provided that the original work is properly attributed. 


\section{Introduction}

Angelman syndrome (AS) is a rare neurodevelopmental disorder characterized by intellectual disability, impaired communication, ataxia, seizures, as well as a happy disposition with a high degree of excitability, smiling, and easily provoked laughter (Williams and Franco, 2010; Bird, 2014). AS is caused by dysfunction of maternal ubiquitin protein ligase E3A (UBE3A), typically from a de novo deletion in the 15q11-q13 region (Albrecht et al., 1997). Restoring functional UBE3A is seemingly possible by innovative gene therapy approaches, including antisense oligonucleotides (Meng et al., 2015), viral vector delivery (Daily et al., 2011), artificial transcription factors (Bailus et al., 2016), stem cell-mediated therapies (Adhikari et al., 2021), and the cutting edge Cas9 (Wolter et al., 2020). Gene replacement therapy is therefore on the horizon for AS; and indeed, two clinical trials using "gene therapy-like" antisense oligonucleotide interventions began recruitment in 2020 (GeneTx NCT04259281; Roche NCT04428281).

Indispensable to such a strategy of therapeutic development are in vivo studies using preclinical model systems with rigorous translational outcomes. One domain that is critically impaired in AS and other neurodevelopmental disorders but difficult to study in preclinical models because of their lack of human-interpretable language is communication. The increasing availability of rat models of neurodevelopmental disorders opens up new opportunities to develop preclinical outcome measures of social communication. While the mouse has been the preferred model species in recent decades because of the genetic technologies available, there are complex behaviors and physiological processes difficult or impossible to investigate in mice that are easily observable in rats (Hofer et al., 2002; Portfors, 2007; Hammerschmidt et al., 2012; Wöhr and Schwarting, 2013; Portfors and Perkel, 2014; Ellenbroek and Youn, 2016).

One prominent example is the greater sophistication and complexity in the rat acoustic communication system. While both mice and rats emit ultrasonic vocalizations (USVs), rats emit USVs that serve as situation-dependent, evolved signals which accomplish important communicative functions that are not observed as functions of mouse USV, such as low-frequency $22 \mathrm{kHz}$ "alarm calls," which rats use to warn of potential threats (Blanchard et al., 1991; Wöhr and Schwarting, 2007, 2013; Sadananda et al., 2008; Brudzynski, 2013; Kisko et al., 2017; Fendt et al., 2018). The recent generation of the first rat model of AS therefore provides the unique opportunity to study a greater diversity of social and communication behaviors compared with those previously available in mouse models (Jiang et al., 2010; Huang et al., 2013; Parker et al., 2014; Ellenbroek and Youn, 2016; HaronyNicolas, 2017; Homberg et al., 2017; Kisko et al., 2018; Kondrakiewicz et al., 2019; Dutta and Crawley, 2020; Netser et al., 2020; Reppucci et al., 2020).

To build on the initial reports describing the Ube3a deletion rat model of AS, which revealed deficits in motor, cognition, social approach, and pup vocalizations (Berg et al., 2020c; Dodge et al., 2020), we sought to investigate nuanced social behaviors and further characterize vocalization patterns. With numerous novel therapies being assessed in clinical trials and at the investigational drug discovery level, AS-relevant outcome measures are vital for demonstrating functional efficacy of the varied intervention approaches. Leveraging the rat's social communication system, we discovered that the Ube3a maternal deletion rat $\left(\right.$ Ube $3 a^{\text {mat-/pat }+}$ ) produced excessive signals of positive affect characteristic of AS. Several other AS-relevant phenotypes were evident, including atypical social interactions and maladaptive impairments in gait and cognition. We also identified reduced hippocampal long-term potentiation (LTP), observed in mouse models of AS but not yet in rats, as a putative cellular mechanism underlying the learning and memory deficits apparent in the model. Finally, our neuroimaging analysis revealed decreased brain volume and pronounced increasing severity with age.

\section{Materials and Methods}

\section{Subjects}

Subjects were male and female Sprague Dawley Ube $3 a^{\text {mat-/pat }}{ }^{+}$rats and their WT littermates $\left(U_{b e} 3 a^{\text {mat }+/ \text { pat }^{+}}\right.$) generated from breeding pairs of paternal Ube3a deletion females and WT males purchased from Envigo. The initial generation of Ube3a deletion rats using CRISPR/Cas9 was described previously (Berg et al., 2020c). Genotyping was performed using a small sample of tail tissue collected at postnatal day (PND) 2, REDExtract-N-Amp (Sigma Aldrich), and primers Rube1123 TAGT GCTGAGGCACTGGTTCAGAGC, Rube1606r TGCAAGGGGTAGCT TACTCATAGC, Ub3aDelSpcfcF6 ACCTAGCCCAAAGCCATCTC, and Ub3aDelR2 GGGAACAGCAAAAGACATGG. All animals were socially housed in a temperature-controlled vivarium maintained on a 12:12 light-dark cycle with testing occurring during the light phase. All procedures were conducted in compliance with the National Institutes of Health's Guide for the care and use of laboratory animals and approved by the Institutional Animal Care and Use Committee of the University of California Davis. To minimize the carryover effects from repeated testing and handling, seven mixed-sex cohorts of rats were tested and behavioral tests were conducted in order of least to most stressful with at least $48 \mathrm{~h}$ break between tests. Each cohort was comprised of 4-9 litters, and subjects were sampled as follows: subjects for $50 \mathrm{kHz}$ USV playback were sampled from Cohort 1; contextual and cued fear conditioning from Cohort 2; gait analysis, heterospecific play, and social play from Cohort 3; acoustic startle and LTP from Cohort 4; spontaneous exploratory USV from Cohort 5; spontaneous alternation from Cohort 6; and olfactory discrimination from Cohort 7. Following behavioral testing, rats from Cohort 3 were perfused for MRI.

\section{Juvenile USV in response to heterospecific play}

At PND 30 to PND 34, rats were provided daily heterospecific play sessions involving manual stimulation using a slightly abbreviated procedure from those described previously (Burgdorf and Panksepp, 2001; Schwarting et al., 2007; Wöhr et al., 2009). For 5 min on 5 consecutive days, rats were individually manipulated by a familiar experimenter using a single clean hand within a clean, empty version of the home cage with fresh bedding $(37.2 \mathrm{~cm}$ [length] $\times 30.8 \mathrm{~cm}$ [width] $\times 18.7 \mathrm{~cm}$ [height]; illuminated to $\sim 30$ lux) while vocalizations were recorded with an overhead ultrasonic microphone (Avisoft Bioacoustics) for later scoring by a trained observer blinded to genotype. The number of calls emitted during each $30 \mathrm{~s}$ interval were counted and classified as either high $(50 \mathrm{kHz}$ ) or low (short $22 \mathrm{kHz}$ ) frequency using a threshold of $33 \mathrm{kHz}$. Calls emitted during the minute immediately preceding the heterospecific play sessions on days 2-4 ("anticipation") were also counted and classified.

All rats were handled by the experimenter in a standardized fashion ( 5 min on $3 \mathrm{~d}$ ) before the first heterospecific play session. The physical manipulations performed during heterospecific play were tickling the subject's neck $(2 \times)$, tickling the subject's belly $(1 \times)$, pushing into their shoulders ("push and drill"; $1 \times$ ), and flipping the subject onto their back and momentarily pinning them down ("flip over"; $3 \times$ ). Each manipulation lasted $30 \mathrm{~s}$ with three $30 \mathrm{~s}$ breaks interspersed at 0,60 , and $150 \mathrm{~s}$, during which the experimenter did not initiate touching the subject but moved their hand around the cage to encourage following or chasing. To provide a standardized experience, a single experimenter conducted the procedure for all subjects and the experimenter remained unaware of USV being emitted during the test, performing the manipulations in an equivalent manner for all rats. To mitigate any potential effect of order, the sequence of manipulations was reordered each day but 
remained consistent across all animals. The testing order of the subjects was also changed from day to day.

\section{Juvenile spontaneous exploratory USV}

At PND 30, rats were individually placed in a clean, empty version of the home cage (illuminated to $\sim 30$ lux) with clean bedding for $5 \mathrm{~min}$ similarly to methods described previously (Schwarting et al., 2007; Wöhr et al., 2008). Recording of USVs began immediately following the subject being placed into the cage, and no other animals or any experimenter were present in the room during recording. Calls were classified by a trained observer blinded to genotype as either high $(50 \mathrm{kHz})$ or low (short $22 \mathrm{kHz}$ ) frequency using a threshold of $33 \mathrm{kHz}$.

\section{Juvenile USV in response to playback of $50 \mathrm{kHz} U S \mathrm{~V}$}

At PND $30 \pm 4$, subjects were individually presented with 1 min of natural prosocial $50 \mathrm{kHz}$ USV while on a radial maze illuminated to $\sim 8$ lux as described previously (Berg et al., 2018, 2020c). USVs were presented to individual subjects using an established playback paradigm (Wöhr et al., 2016; Berg et al., 2018), including the USV stimulus previously demonstrated to elicit social approach (behavior shown in Berg et al., 2020c). The USV stimulus consisted of 221 natural $50 \mathrm{kHz}$ USV recorded from a naive male rat during exploration of a cage containing a recently separated cage mate. A $3.5 \mathrm{~s}$ sequence of 13 calls was repeated 17 times such that $22150 \mathrm{kHz}$ calls were presented within $1 \mathrm{~min}$. Response vocalizations were recorded with an overhead ultrasonic microphone (Avisoft Bioacoustics), and the number of calls emitted during the minute of playback were counted by a trained observer blinded to genotype and classified as high $(50 \mathrm{kHz})$ or low (short $22 \mathrm{kHz}$ ) frequency using a threshold of $33 \mathrm{kHz}$.

\section{Juvenile social play}

At PND $38 \pm 1$, social play behavior was assessed following a protocol described previously (Berg et al., 2018, 2020a,b). Each subject rat was placed with a freely moving, unfamiliar, strain-, sex-, and age-matched WT stimulus rat for $10 \mathrm{~min}$ in a clean, empty test arena (illuminated to $\sim 30$ lux) containing a thin layer of clean bedding. In order to facilitate social play, each subject and stimulus animal was socially isolated in a separate holding room for $30 \mathrm{~min}$ before the test. Stimulus animals were generated from WT Sprague Dawley breeders (Envigo) and handled in a standardized manner ( $5 \mathrm{~min}$ on $3 \mathrm{~d}$ ) before the assay. The interaction was video-recorded, and behaviors were later scored by a trained observer blinded to genotype as follows: Social sniffing: sniffing the stimulus rat's face, body, or tail; Anogenital sniffing: sniffing the stimulus rat's anogenital region; Self-grooming: subject grooming itself; Exploring: sitting, walking, rearing, or sniffing the ground or wall; Following or chasing: following (walking pace) or chasing (running pace) the stimulus rat; Rough-and-tumble playing: accelerated movement involving chasing, pouncing, pinning, tumbling, and/or boxing which requires the stimulus rat's participation (i.e., reciprocity); Push past: directed movement toward the stimulus rat to get next to, or move closely past, without sniffing or otherwise engaging; Push under or crawl over: head dip under the stimulus rat's belly or completely stepping over the stimulus rat; and Pounce: both paws placed via leap or directed movement onto the stimulus rat's back. Blind scoring was possible since Ube $3 a^{\text {mat-/pat+ }}$ rats have normal body weight and are physically indistinguishable from their WT littermates (Berg et al., 2020c).

\section{Olfactory discrimination}

At PND $42 \pm 3$, the ability of rats to discriminate between a social and nonsocial odor was tested by measuring the time spent investigating odor-saturated cotton swabs. Subjects were individually tested in clean chambers $(40.6 \mathrm{~cm}$ [length] $\times 40.6 \mathrm{~cm}$ [width] $\times 28 \mathrm{~cm}$ [height] $)$ dimly illuminated to $\sim 30$ lux. On the day before the test, rats were habituated to the test chamber containing a clean dry cotton swab $(15.2 \mathrm{~cm} \mathrm{l})$ for $20 \mathrm{~min}$. The tip of the swab was secured $3 \mathrm{~cm}$ above the floor in the center of the arena by being attached to the top of a clean weighted glass dome $(7.6 \mathrm{~cm}$ [diameter] $\times 10 \mathrm{~cm}$ [height] $)$ and angled downward. On the day of the test, rats were again habituated to the arena containing a clean dry cotton swab for $10 \mathrm{~min}$, followed by a swab soaked in water, then vanilla (1:100 dilution; McCormick), and then a social scent. The social scent was collected by wiping a cotton swab in a zig zag pattern along the bottom of a cage of same sex but unfamiliar Sprague Dawley rats (Envigo). Each saturated swab was presented for $2 \mathrm{~min}$, and the order of odor presentation was consistent across all animals. Time spent sniffing the swab soaked with vanilla scent and the swab soaked with social scent (defined as the nose within $2 \mathrm{~cm}$ of the cotton swab tip) was measured using videotracking software (EthoVision XT, Noldus Information Technology), which was subsequently validated manually.

\section{Juvenile gait}

At PND 25, gait metrics were collected using the DigiGait automated treadmill system and analysis software (Mouse Specifics). Subjects were placed individually into the enclosed treadmill chamber and allowed to acclimate before the belt was turned on. The belt speed was slowly increased to a constant speed of $20 \mathrm{~cm} / \mathrm{s}$, at which each rat was recorded making clearly visible consecutive strides for 3-6 s.

\section{Juvenile contextual and cued fear conditioning}

At PND $43 \pm 1$, learning and memory were assessed using an automated fear conditioning chamber (Med Associates) following methods previously described (Copping et al., 2017; Adhikari et al., 2019; Berg et al., $2020 \mathrm{~b}$ ). On day 1 , rats were trained via exposure to a series of three noise-shock (conditioned stimulus-unconditioned stimulus; CS-US; $80 \mathrm{~dB}$ white noise, $0.7 \mathrm{~mA}$ foot shock) pairings inside a sound-attenuated chamber with specific visual, tactile, and odor cues. On day 2, contextual memory was tested by placing each subject back inside the training environment (no noise or foot shock occurred). On day 3, cued memory was evaluated by placing subjects into a novel context with altered visual, tactile, and odor cues. Following a period of exploration, the white noise CS was presented for $3 \mathrm{~min}$. Time spent freezing was measured using VideoFreeze software (Med Associates).

\section{Prepulse inhibition of an acoustic startle response}

At 9-10 weeks of age, prepulse inhibition was measured using a SR-Lab System (San Diego Instruments). Subjects were placed in a clear plastic cylinder, which was mounted onto a platform connected to piezoelectric transducers inside a sound-attenuating chamber with internal speakers. The background noise level in the chamber was $70 \mathrm{~dB}$ white noise. Each session consisted of a 5 min acclimation period followed by a pseudorandomized presentation of 50 trials of five different trial types: one trial type was a $40 \mathrm{~ms} 120 \mathrm{~dB}$ startle stimulus, three trial types involved an acoustic prepulse $(74,82$, or $90 \mathrm{~dB})$ presented $120 \mathrm{~ms}$ before the $120 \mathrm{~dB}$ startle stimulus, and there were also trials with no startle stimulus to measure baseline movement inside the cylinder. Each trial type was presented in 10 blocks and was randomized within blocks. The intertrial interval varied randomly between 10 and $20 \mathrm{~s}$. Percent PPI was calculated using the equation: \% PPI $=[1-($ Prepulse $/$ Max Startle $)] \times 100$.

\section{Spontaneous alternation}

At 10 weeks of age, spontaneous alternation was measured by allowing rats to freely explore a novel Y-maze (black, opaque; arms: $53.3 \mathrm{~cm}$ [length] $\times 11.4 \mathrm{~cm}$ [width] $\times 27.9 \mathrm{~cm}$ [height]; illuminated to $\sim 30$ lux) for $8 \mathrm{~min}$. An overhead camera connected to videotracking software (EthoVision XT; Noldus Information Technology) was used to quantify the number of arm entries, the number of errors (defined as the sum of direct and indirect revisits to an arm), the number of spontaneous alternations (defined as consecutively visiting all three arms without any revisit), and the maximum number of possible alternations for the entire session.

\section{LTP}

Acute slice preparation. At 12-13 weeks of age, subjects were deeply anesthetized with isoflurane; and following decapitation, the brain was rapidly removed and submerged in ice-cold, oxygenated $\left(95 \% \mathrm{O}_{2} / 5 \%\right.$ $\mathrm{CO}_{2}$ ) ACSF containing the following (in $\mathrm{mm}$ ): $124 \mathrm{NaCl}, 4 \mathrm{KCl}, 25$ $\mathrm{NaHCO}_{3}, 1 \mathrm{NaH}_{2} \mathrm{PO}_{4}, 2 \mathrm{CaCl}_{2}, 1.2 \mathrm{MgSO}_{4}$, and 10 glucose. On an icecold plate, the brain hemispheres were separated, blocked, and the hippocampi removed. The $400-\mu \mathrm{m}$-thick slices were then cut using a McIlwain tissue chopper (Brinkman). Slices from the dorsal third of the hippocampus were used. Slices were incubated at $33^{\circ} \mathrm{C}$ for $20 \mathrm{~min}$ and 
then maintained in submerged-type chambers that were continuously perfused (2-3 $\mathrm{ml} / \mathrm{min})$ with ACSF and allowed to recover for at least 1.5$2 \mathrm{~h}$ before recordings. Just before start of experiments, slices were transferred to a submersion chamber on an upright Olympus microscope, perfused with $30.4^{\circ} \mathrm{C}$ normal ACSF saturated with $95 \% \mathrm{O}_{2} / 5 \% \mathrm{CO}_{2}$.

Electrophysiological recordings. A bipolar, nichrome wire stimulating electrode (MicroProbes) was placed in stratum radiatum of the CA1 region and used to activate Schaffer collateral/commissural fiber synapses. Evoked fEPSPs (basal stimulation rate $=0.033 \mathrm{~Hz}$ ) were recorded in stratum radiatum using borosilicate pipettes (Sutter Instruments) filled with ACSF (resistance 5-10 M $\Omega$ ). Submerged-type recording chambers were used for all recordings. All recordings were obtained with a MultiClamp 700B amplifier (Molecular Devices), filtered at $2 \mathrm{kHz}$, and digitized at $10 \mathrm{~Hz}$. To determine response parameters of excitatory synapses, basal synaptic strength was quantified by comparing the amplitudes of presynaptic fiber volleys and postsynaptic fEPSP slopes for responses elicited by different intensities of SC fiber stimulation. Presynaptic neurotransmitter release probability was compared by paired-pulse facilitation experiments, performed at 25, 50, 100, and 250 ms stimulation intervals. LTP was induced by high-frequency stimulation (HFS) using a $2 \times$ tetanus (1-s-long train of $100 \mathrm{~Hz}$ stimulation) with a $10 \mathrm{~s}$ intertetanus interval. At the start of each experiment, the maximal fEPSP amplitude was determined and the intensity of presynaptic fiber stimulation was adjusted to evoke fEPSPs with an amplitude $\sim 40 \%-50 \%$ of the maximal amplitude. The average slope of EPSPs elicited 55-60 min after HFS (normalized to baseline) was used for statistical comparisons.

\section{MRI}

At 6.5 months of age, ex vivo neuroimaging was conducted by following a protocol previously described (Berg et al., 2018, 2020c). Brains were flushed and fixed via transcardial perfusion with $50 \mathrm{ml}$ PBS containing $10 \mathrm{U} / \mathrm{ml}$ heparin and $2 \mathrm{~mm}$ ProHance (gadolinium contrast agent; Bracco Diagnostics) followed by $50 \mathrm{ml}$ 4\% PFA in PBS containing $2 \mathrm{~mm}$ ProHance. Brains were incubated in the $4 \%$ PFA solution at $4^{\circ} \mathrm{C}$ for 24 $\mathrm{h}$, transferred to a $0.02 \%$ sodium azide PBS solution, and then incubated at $4^{\circ} \mathrm{C}$ for at least 1 month before being scanned. MRI of the brains within their skulls was conducted using a multichannel 7.0 Tesla scanner (Agilent Technologies). Seven custom millipede coils were used to image the brains in parallel (Bock et al., 2005; Lerch et al., 2011). Parameters used in the anatomic MRI scans are as follows: T2-weighted 3D fast spin echo sequence, with a cylindrical acquisition of $\mathrm{k}$-space, and with a TR of $350 \mathrm{~ms}$, and TEs of $10.5 \mathrm{~ms}$ per echo for 12 echoes, FOV $36 \times 36 \times 40$ $\mathrm{mm}^{3}$, and a matrix size of $456 \times 456 \times 504$, giving an image with 0.079 $\mathrm{mm}$ isotropic voxels (Spencer Noakes et al., 2017). The current scan time for this sequence is $\sim 3 \mathrm{~h}$.

To visualize and compare any changes in the rat brains, the images were linearly and nonlinearly registered together using the pydpiper framework. Registrations were performed using a combination of mni_autoreg tools (Collins et al., 1994) and ANTS (advanced normalization tools) (Avants et al., 2011). Following registration, a population atlas was created representing the average anatomy of the study sample. At the end of the registration process, all the scans were deformed into alignment with one another in an unbiased fashion. This allows for analysis of the deformations required to register the brains together, which can be used to assess the volume of the individual brains and compared them to one another (Bishop et al., 2006; Lerch et al., 2008a,b,c; Nieman et al., 2010, 2018). For comparisons to the juvenile brains, a separate registration pipeline was used that included all the brains from this study as well as the previous Berg et al. (2020c) study. Volumetric differences were calculated on a regional and a voxelwise basis. An in-house manually segmented hierarchical rat brain atlas was used to calculate the volumes of 52 different segmented structures. These structures were derived from multiple atlases (Dorr et al., 2008; Steadman et al., 2014) and then modified for use in the rat brain.

Experimental design and statistical analyses

Statistical analyses were performed using GraphPad Prism 8 statistical software (GraphPad Software). Clampex 10.6 software suite (Molecular
Devices) was used for analyzing electrophysiological data. Congruent with previous studies, no significant sex differences were detected, so the results herein include both males and females. Effect sizes and power were determined using Cohen's $d$.

Analysis of behavior and LTP. For single comparisons between two groups, either a Student's $t$ test or Mann-Whitney $U$ test was used. Data that passed distribution normality tests, were collected using continuous variables, and had similar variances across groups were analyzed via Student's $t$ test. Alternatively, a Mann-Whitney $U$ test was used. Either a two-way ANOVA or two-way repeated-measures ANOVA was used to analyze the effects of genotype and a second factor. In repeated-measures ANOVA, genotype was the between-group factor and time, limb set, test phase, scent, or prepulse intensity was the within-group factor. Post hoc comparisons were performed following a significant main effect or interaction and were conducted using Holm-Sidak's multiple comparisons test controlling for multiple comparisons. Data points within 2 SDs of the mean were included, all significance tests were two-tailed, and a $p$ value of $<0.05$ was considered significant.

Analysis of MRI. Statistical analyses were used to compare both the absolute and relative volumes voxelwise as well as across the 52 different hierarchical structures in the rat brains. Absolute volume was calculated as $\mathrm{mm}^{3}$, and relative volume was assessed as a measure of $\%$ total brain volume. Voxelwise and regional differences were assessed using linear models. All image analysis tools and software are available on Github (https://github.com/Mouse-Imaging-Centre). Multiple comparisons were controlled for using the false discovery rate (Genovese et al., 2002).

\section{Results}

\section{Overabundant emission of laughter-like $50 \mathrm{kHz}$ calls in juvenile $U b e 3 a^{\text {mat-/pat }+}$ rats}

Since abnormal expressive communication and elevated rates of positive affect are key clinical features of AS, we sought to quantify these characteristics in Ube $3 a^{\text {mat-/pat }+}$ and Ube $3 a^{\text {mat }+/ \text { pat }+}$ (WT) rats. While vocalizations are readily collected during social play, recording USVs from multiple interacting animals makes it difficult to determine which animal made each call. We therefore took advantage of the fact that rats emit laughter-like $50 \mathrm{kHz}$ calls when social play is simulated by an experimenter via tickling and other physical maneuverings (Burgdorf and Panksepp, 2001; Burgdorf et al., 2005, 2008; Ishiyama and Brecht, 2016). We implemented a standardized heterospecific play procedure (Fig. $1 A$ ) to elicit USVs (Fig. $1 B$ ) while maintaining full confidence in the identity of the caller and controlling for the level of physical interaction across subjects.

We discovered that, while both groups increased $50 \mathrm{kHz}$ USV emission across consecutive sessions, Ube $3 a^{\text {mat-/pat+ }}{ }^{+}$emitted a substantially elevated level of $50 \mathrm{kHz}$ USVs (Fig. $1 C$; $F_{\text {Genotype(G) }}(1,48)=$ 7.351, $p=0.009 ; \quad F_{\mathrm{Day}(\mathrm{D})}(3.007,144.3)=10.82, \quad p<0.0001$; $\left.F_{\mathrm{D} \times \mathrm{G}}(4192)=1.052, p>0.05\right)$. In total, Ube $3 a^{\text {mat-/pat }+}$ emitted an average of $33 \pm 5$ USVs per minute (mean \pm SEM), more than twice the rate of controls, which produced an average of $15 \pm 3$ calls per minute (Fig. $1 D ; U=175, p=0.007, d=0.77$ ). A closer examination revealed that $50 \mathrm{kHz}$ USVs were elevated during the break and belly tickle phases (Fig. $1 E ; F_{\mathrm{G}}(1,48)=$ 6.927, $p=0.011 ; F_{\text {Phase }(\mathrm{P})}(1.722,82.64)=27.83, p<0.0001$; $F_{\mathrm{P} \times \mathrm{G}}(4192)=2.075, p>0.05$; post hoc: break, $p=0.023, d=0.85$; belly tickle, $p=0.023, d=0.84$ ), although calling during the other phases also trended higher, providing strong evidence of elevated positive affect and a high hedonic impact of the assay (neck tickle, $p=0.057, d=0.62$; push and drill, $p=0.057, d=0.65$; flip over, $p=0.057, d=0.69)$. There was no effect of sex, nor an interaction with sex $(p>0.05)$, for any parameter.

Additionally, $50 \mathrm{kHz}$ USVs were more frequently emitted during the anticipation period immediately before the play 
A

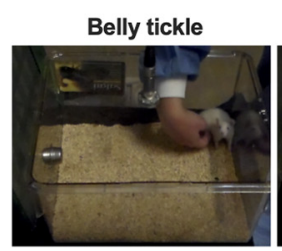

Neck tickle

Push and drill

Flip over
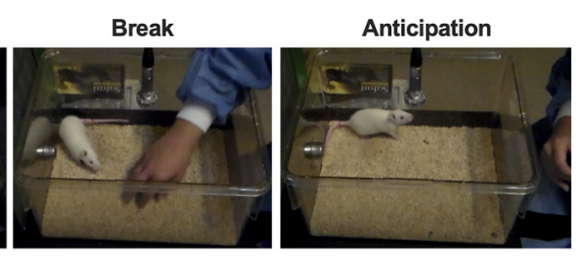

B
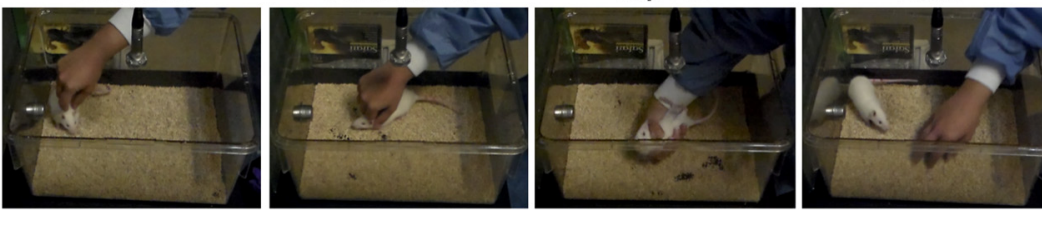

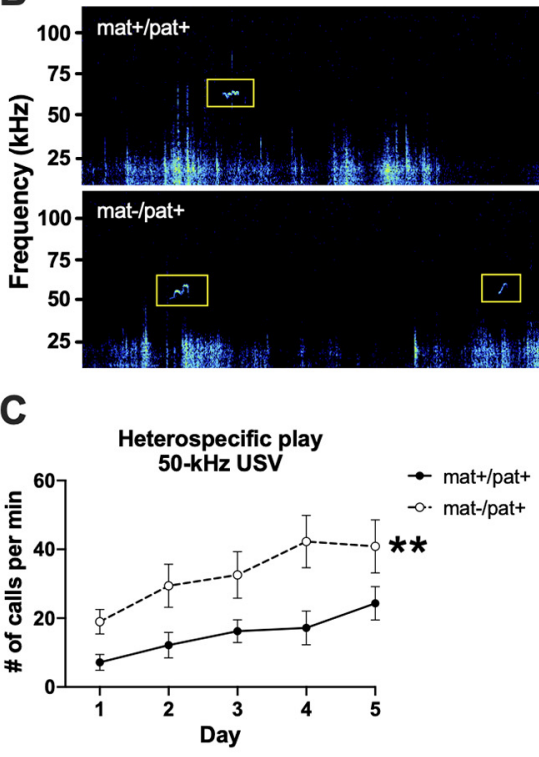

F
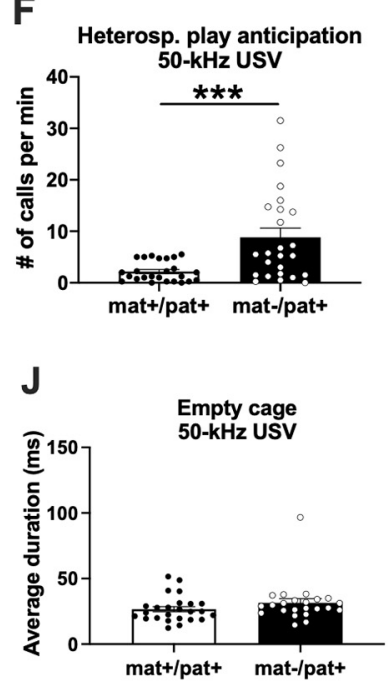

$\mathbf{N}$

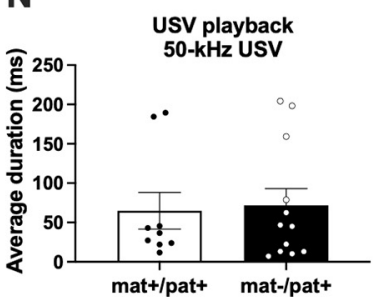

G

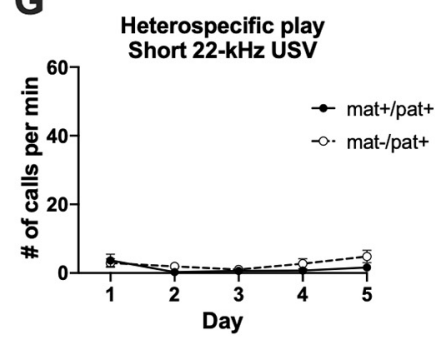

K

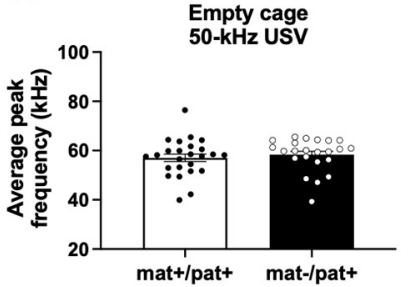

0

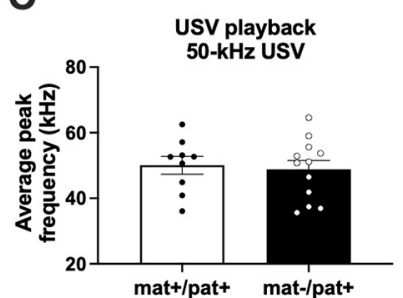

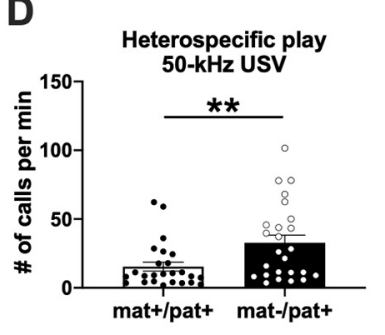

E

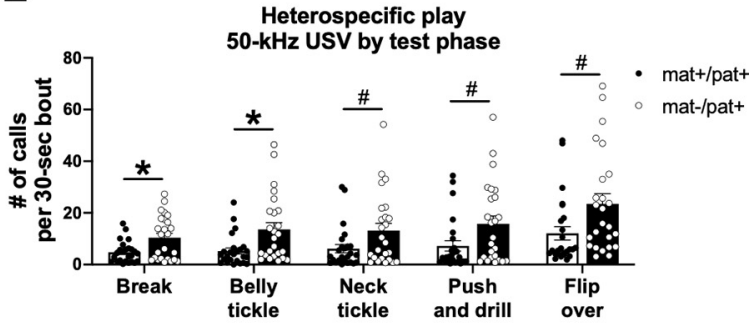

H
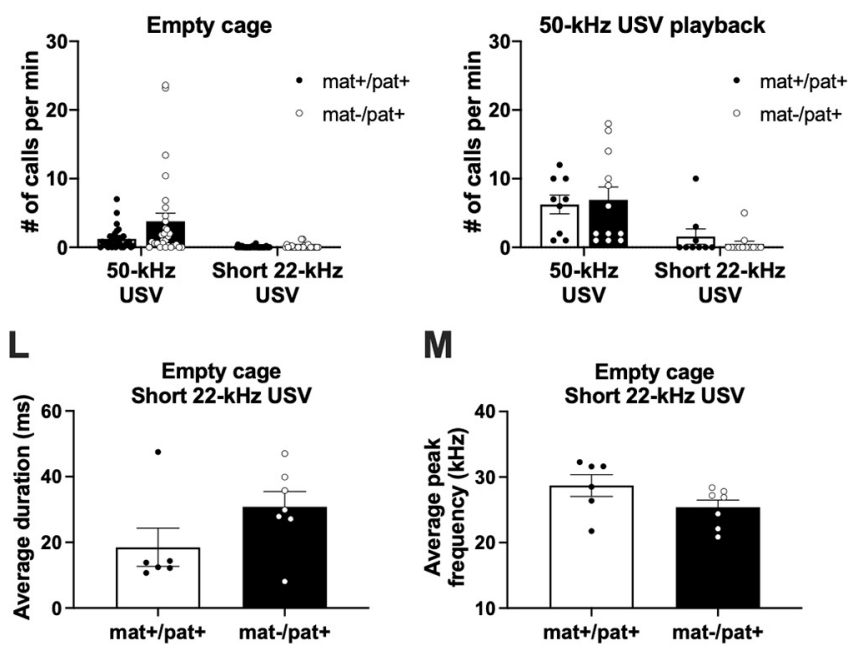

P

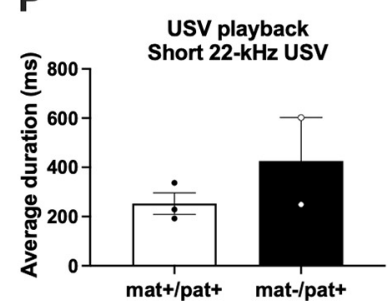

Q

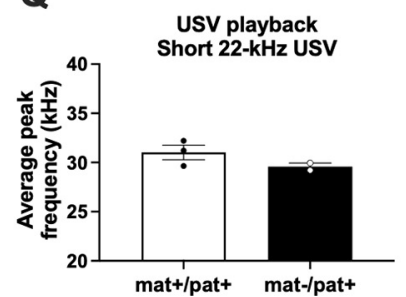

Figure 1. Overabundant emission of laughter-like $50 \mathrm{kHz}$ calls in juvenile Ube3a ${ }^{\text {mat-/pat }+}$ rats. $\boldsymbol{A}$, Example images of the manipulations used to mimic social play and elicit USVs. $\boldsymbol{B}$, Example spectrograms of USVs from a WT littermate control (Ube3a ${ }^{\text {mat }+/ \text { pat }+}$; mat +/pat +; top) and Ube $3 a^{\text {mat-/pat }+}$ rat (mat-/pat+; bottom). C, Across 5 d of heterospecific play sessions, 50 kHz USV emission increased with repeated testing in both mat-/pat $+(n=25)$ and controls $(n=25)$, but the emission rate was substantially elevated in mat-/pat + . $\boldsymbol{D}, 0 \mathrm{n}$ average, mat-/pat + rats produced $50 \mathrm{kHz}$ USVs at more than twice the rate of controls. $E$, Specifically, $50 \mathrm{kHz}$ calling was abnormally high during the break and belly tickle phases, with trending increases during neck tickle, push and drill, and flip over. $\boldsymbol{F}$, Before the onset of play, mat-/pat + rats emitted anticipatory $50 \mathrm{kHz}$ USVs at $>3$ times the rate of controls. $\boldsymbol{G}$, Production of short $22 \mathrm{kHz}$ USVs was low, did not differ between genotypes, and did not change over subsequent play sessions. $\boldsymbol{H}$, The rates of $50 \mathrm{kHz}$ and short $22 \mathrm{kHz}$ calling during empty cage exploration were comparable between genotypes (mat $+/ \mathrm{pat}+, n=32$; mat-/pat,$+ n=29$ ), as were the $(I) 50 \mathrm{kHz}$ and short $22 \mathrm{kHz}$ calling rates in response to hearing playback of conspecific $50 \mathrm{kHz}$ USV 
A

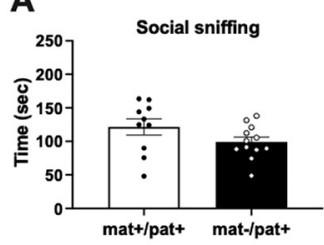

$\mathbf{F}$

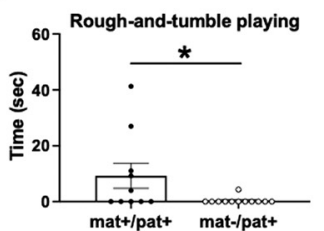

B

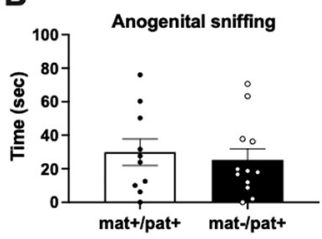

G

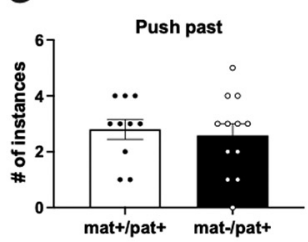

C

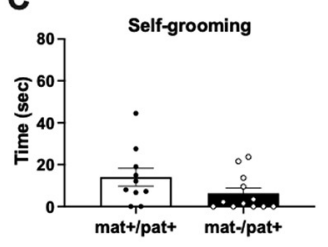

H

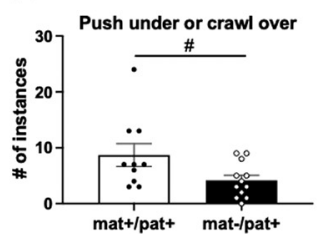

D

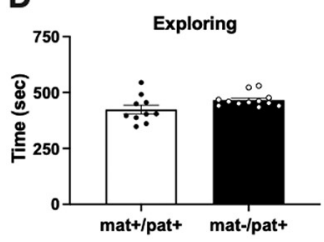

I

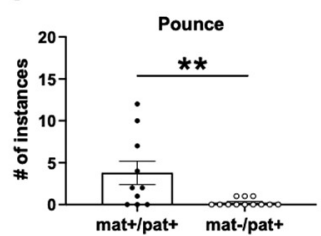

E

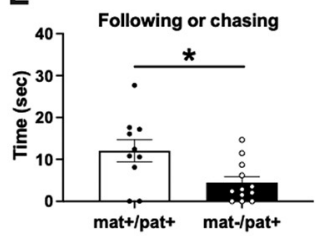

$\mathbf{J}$

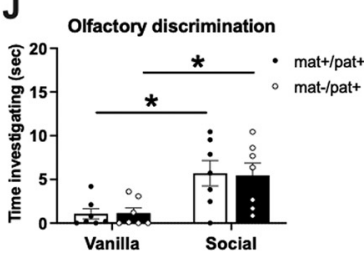

Figure 2. Intact social interest but deficient expression of key social interaction behaviors in juvenile Ube3amat-/pat+ rats. $\boldsymbol{A}$, During a 10 min interaction session with a novel same-sex WT conspecific, Ube $3 a^{\text {mat-/pat+ }}$ rats (mat-/pat $\left.+; n=12\right)$ spent similar amounts of time social sniffing, $(\boldsymbol{B})$ anogenital sniffing, $(\boldsymbol{C})$ self-grooming, and $(\boldsymbol{D})$ exploring the arena compared with WT littermate controls (Ube3a ${ }^{\text {mat }+/ \text { pat }+} ;$ mat $+/$ pat $+; n=10$ ). $\boldsymbol{E}$, Robust deficits, however, were discovered in the time spent following or chasing and $(\boldsymbol{F})$ rough-and-tumble playing. $\boldsymbol{G}$, The number of push pasts were similar across genotypes, but $(\boldsymbol{H})$ there was a trend for mat-/pat + to less frequently push under or crawl over and $(\boldsymbol{I})$ mat-/pat + rats did not perform nearly as many pounces as WT littermates. $J$, A separate test of olfactory discrimination revealed normal sniff times of social and nonsocial scents. Time spent investigating novel odors was similar for mat-/pat $+(n=7)$ and mat $+/$ pat + rats $(n=7)$ and both groups spent more time investigating a social scent compared with a non-social vanilla odor. Data are mean \pm SEM. $\boldsymbol{E}-\boldsymbol{I},{ }^{* *} p<0.01,{ }^{*} p<0.05,{ }^{*} p<0.065$, Mann-Whitney $U$ test. $\boldsymbol{J},{ }^{*} p<0.05$, repeated-measures ANOVA, Holm-Sidak's post hoc.

sessions (Fig. $1 F ; U=146.5, p=0.001, d=1.04$ ). In total, across all four anticipation time points (days 2-5), Ube $3 a^{\text {mat-/pat }+}$ emitted an average of $9 \pm 2$ USVs per minute (mean \pm SEM), $>4$ times the rate of WTs, which produced an average of $2 \pm 0.4$ calls per minute. This indicates that $U b e 3 a^{\text {mat-/pat+ }}$ predicted the impending onset of play and that the interaction had a high degree of incentive salience.

Excessive vocalization by $U b e 3 a^{\text {mat-/pat }+}$ rats was specific to $50 \mathrm{kHz}$ USVs. Production of short $22 \mathrm{kHz}$ USV, which are emitted in modest amounts during play, was low and did not differ between genotypes (Fig. $1 G ; F_{\mathrm{G}}(1,48)=1.771, p>0.05$; $\left.F_{\mathrm{D}}(1.825,87.62)=3.160, p>0.05 ; F_{\mathrm{D} \times \mathrm{G}}(4192)=1.330, p>0.05\right)$. Elevated $50 \mathrm{kHz}$ calling by $U b e 3 a^{\text {mat-/pat }+}$ was also specific to being provoked by heterospecific play, as $50 \mathrm{kHz}$ and short 22 $\mathrm{kHz}$ USV production was normal during exploration of an empty cage (albeit a slight trend toward more $50 \mathrm{kHz}$ USV; Fig. $1 H ; 50 \mathrm{kHz}, U=374.5, p>0.05 ; 22 \mathrm{kHz}, U=433, p>0.05$, $d=0.56$ ) and in response to the acoustic presentation of $50 \mathrm{kHz}$ USVs (Fig. 1I; $50 \mathrm{kHz}, U=53, p>0.05 ; 22 \mathrm{kHz}, U=44.50$, $p>0.05)$. No gross abnormalities in call structure were observed. Specifically, $50 \mathrm{kHz}$ calls were of normal duration and peak frequency (Fig. 1J: $U=205, p>0.05$; Fig. $1 K: U=240, p>0.05$; Fig. $1 N: U=52, p>0.05$; Fig. 1O: $\left.t_{(19)}=0.3179, p>0.05\right)$, suggesting that increased heterospecific play $50 \mathrm{kHz}$ call numbers were not

\footnotetext{
(mat+/pat,$+ n=9 ;$ mat-/pat,$+ n=12$ ). Call features did not differ by genotype: J, The average duration and $(\boldsymbol{K})$ peak frequency of spontaneous $50 \mathrm{kHz}$ calls made during exploration of an empty cage were comparable between mat- $/$ pat $+(n=23)$ and mat $+/$ pat + rats $(n=25)$. $\boldsymbol{L}$, The average duration and $(\boldsymbol{M})$ average peak frequency of short $22 \mathrm{kHz}$ calls made within an empty cage were also similar between genotypes (mat $+/$ pat,$+ n=6$; mat-/pat,$+ n=7) . \boldsymbol{N}$, For $50 \mathrm{kHz}$ USVs emitted in response to hearing playback of natural prerecorded $50 \mathrm{kHz}$ rat USVs, average duration and $(\boldsymbol{0})$ average peak frequency were comparable between mat-/pat + rats $(n=12)$ and WT littermates $(n=9)$. $\boldsymbol{P}$, There was no genotype effect on the average duration or $(\boldsymbol{Q})$ average peak frequency of short $22 \mathrm{kHz}$ calls made during USV playback (mat $+/$ pat,$+ n=3$; mat-/pat,$+ n=2$ ). Of note, long $22 \mathrm{kHz}$ USVs known to function as "alarm calls" were very rarely observed, indicating that our paradigms were not aversive. Data are mean \pm SEM. $C$, ${ }^{* *} p<0.01$, repeated-measures ANOVA. $\boldsymbol{D}, \boldsymbol{F},{ }^{* * *} p<0.001,{ }^{* *} p<0.01$, Mann-Whitney $U$ test. $\boldsymbol{E},{ }^{*} p<0.05,{ }^{*} p<0.06$, repeatedmeasures ANOVA, Holm-Sidak's post hoc.
}

inflated by shorter or broken calls. Duration and peak frequency of $22 \mathrm{kHz}$ USVs were also comparable between genotypes (Fig. $1 L: U=12, p>0.05$; Fig. $1 M: t_{(11)}=1.699, p>0.05$; Fig. $1 P$ : $U=1, p>0.05$; Fig. 1Q: $U=1, p>0.05$ ). Since the average duration of the juvenile $22 \mathrm{kHz}$ USV fell short of the usual durations of adult "typical $22 \mathrm{kHz}$ " USVs, we herein refer to them as "short $22 \mathrm{kHz}$ " USVs.

\section{Intact social interest but deficient expression of key social} interaction behaviors in juvenile $U$ be $3 a^{\text {mat-/pat+ }}$ rats We sought to investigate whether elevated $50 \mathrm{kHz}$ USV emission in $U b e 3 a^{\text {mat-/pat+ }}$ rats was associated with greater social engagement with a conspecific. Starting at $\sim 2$ weeks of age, rats play fight with each other by chasing, pouncing, pinning, and wrestling in a manner similar to cats and dogs. Through developmental experience, they learn how to appropriately initiate, engage in, and terminate play bouts with others. In order to more closely examine social behavior and the nuanced reciprocal interactions of social play, we gave juvenile subjects the opportunity to freely interact with a conspecific (Meaney and Stewart, 1981; Panksepp, 1981; Pellis and Pellis, 1998, 2017). Despite emission of more $50 \mathrm{kHz}$ calling during heterospecific play, Ube $3 a^{\text {mat-/pat }+}$ rats showed a normal degree of interest in the stimulus animal, demonstrated by the amounts of time spent social sniffing (Fig. $\left.2 A ; t_{(20)}=1.646, p>0.05\right)$ and anogenital sniffing $\left(\right.$ Fig. $2 B ; t_{(20)}=$ $0.4457, p>0.05)$. Putting forth a similar level of investigative effort suggested that $U b e 3 a^{\text {mat-/pat+ }}$ are just as motivated for social interaction as controls. Levels of self-grooming (Fig. 2C; $U=38$, $p>0.05$ ) and arena exploration (Fig. $2 D ; U=30, p>0.05$ ) were also normal, but $U b e 3 a^{\text {mat-/pat+ }}$ spent markedly less time following or chasing the stimulus rat (Fig. $2 E ; U=29, p=0.041$, $d=1.11$ ). The key observation was the reduced time spent roughand-tumble playing (Fig. $2 F ; U=33.50, p=0.029, d=0.89$ ) compared with WTs. In an attempt to reconcile the near lack of play with intact levels of social interest, we quantified specific components of rough-and-tumble play. While the number of side-to-side social contacts via push pasts were similar across genotypes (Fig. $2 G$; $t_{(20)}=0.3852, p>0.05$ ), there was a trending reduction in the number of push under or crawl overs (Fig. $2 H ; U=31.5, p=0.061, d=0.89)$ and almost a complete lack of 
A

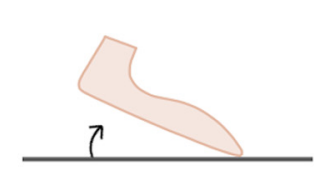

B
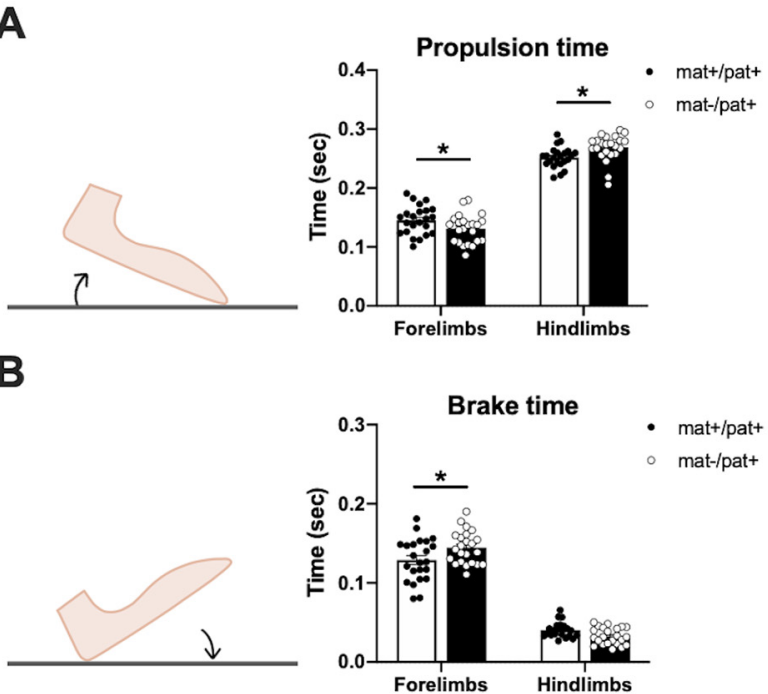

C
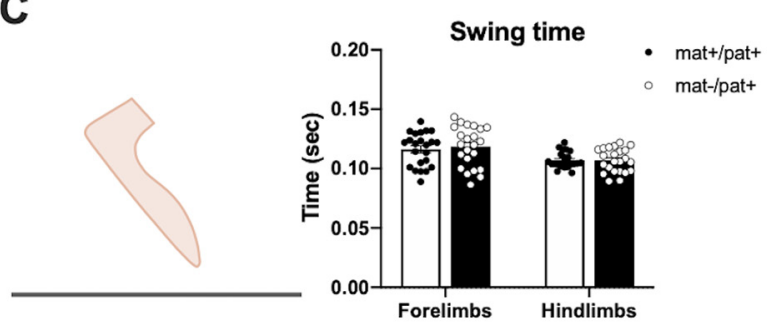

D
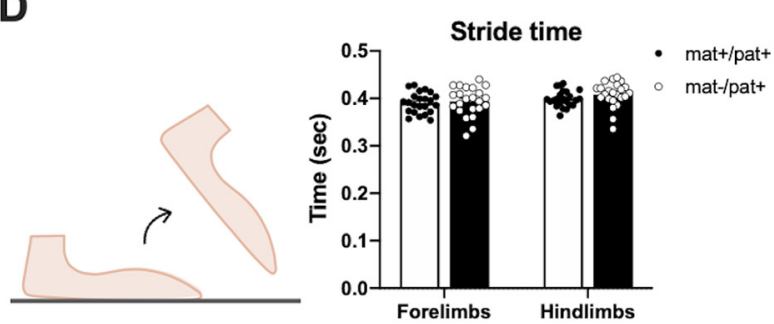

E

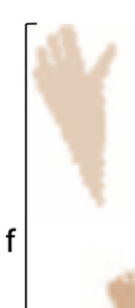

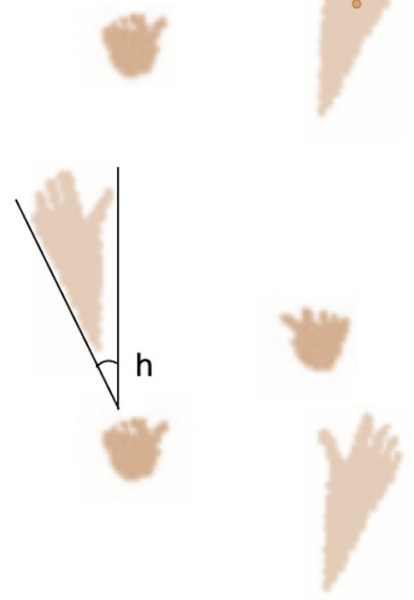

F

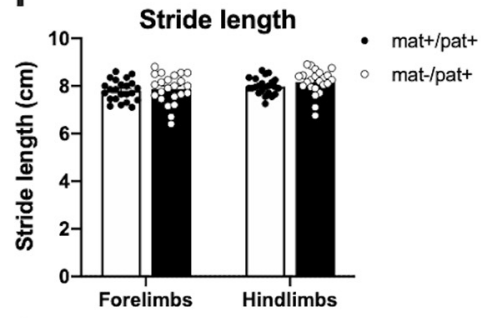

G

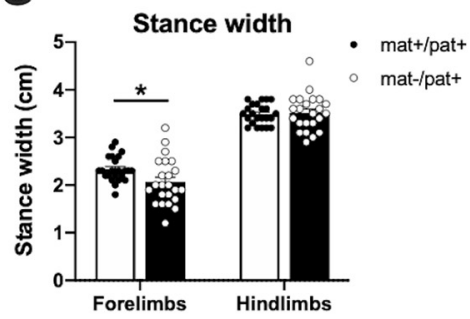

H

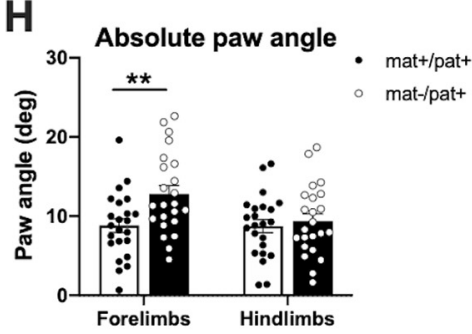

I

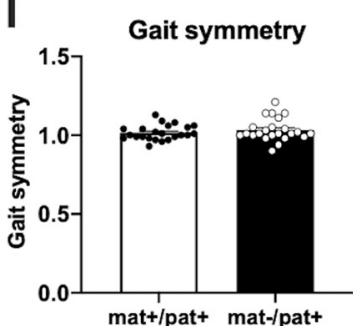

Figure 3. Abnormal gait in Ube3a $a^{\text {mat-/pat }+}$ rats. $A$, While treadmill walking, Ube3a $a^{\text {mat-/pat }+}$ rats (mat-/pat $+; n=23$ ) displayed aberrant propulsion time (time from maximal paw contact with belt to just before liftoff) in both sets of limbs. Compared with WT littermates (Ube $3 a^{\text {mat }+/ \text { pat }+}$; mat $+/$ pat $+; n=23$ ), propulsion time was decreased in the forelimbs and increased in hindlimbs. $\boldsymbol{B}$, Brake time (time from initial to maximal paw contact with belt) was significantly elevated in the forelimbs of mat-/pat + while a trending reduction in hindlimb brake time was found $(p=0.150)$. C, Swing time (no paw contact with the belt) and $(\boldsymbol{D})$ stride time (sum of swing and stance time) were similar across genotypes. $\boldsymbol{E}$, Example paw prints illustrating the spatial gait parameters depicted in $\boldsymbol{F}-\boldsymbol{H}$. $\boldsymbol{F}$, Stride length did not differ between groups, but (G) forelimb stance width was narrower and $(\boldsymbol{H})$ absolute paw angle for the forelimbs was greater, indicating more external rotation in mat-/pat + rats. I, No significant difference in gait symmetry (ratio of forelimb to hindlimb stepping frequency) was detected. Data are mean \pm SEM. ${ }^{* *} p<0.01,{ }^{*} p<0.05$, repeated-measures ANOVA, Holm-Sidak's post hoc.

pouncing in $U b e 3 a^{\text {mat-/pat+ }}$ (Fig. $2 I ; U=24, p=0.008, d=1.14$ ). A separate test of olfaction was used to rule out an olfactory deficit as a confounder of social investigation (Fig. $2 J ; F_{\text {Genotype }(\mathrm{G})}(1,12)=$ $0.0066, p=0.937 ; F_{\text {Scent(S) }}(1,12)=14.20, p=0.003 ; F_{\mathrm{S} \times \mathrm{G}}(1,12)=$ $0.0165, p=0.900$; post hoc: mat+/pat+, $p=0.035$; mat-/pat + , $p=0.035)$.

\section{Abnormal gait in $\mathrm{Ube} 3 a^{\text {mat-/pat+ }}$ rats}

In an effort to assess the potential contribution of motor defects to social play behavior, we explored motor dysfunction, which is a core clinical feature of AS prevalent in mouse models (Huang et al., 2013; Leach and Crawley, 2018) and hypothesized by our group to underlie the open field, rotarod, and marble-burying phenotypes of AS mouse models. Previously, we discovered lower open field vertical activity in Ube $3 a^{\text {mat-/pat }+}$ rats, whereas other activity indices were typical (Berg et al., 2020c). Using the DigiGait automated treadmill system, we found that juvenile
Ube $3 a^{\text {mat-/pat+ }}$ rats displayed robust abnormalities in limb propulsion time, indicating reduced limb strength and less force produced per unit time compared with WTs (Fig. $3 A ; F_{\text {Genotype(G) }}$ $(1,44)=0.0684, p>0.05 ; F_{\operatorname{Limbs}(\mathrm{L})}(1,44)=776.8, p<0.0001$; $F_{\mathrm{L} \times \mathrm{G}}(1,44)=12.80, p<0.001$; post hoc: forelimbs, $p=0.030$, $d=0.60$; hindlimbs, $p=0.022, d=0.85$; Fig. $3 B ; F_{\mathrm{G}}(1,44)=1.012$, $p>0.05, F_{\mathrm{L}}(1,44)=687.0, p<0.0001 ; F_{\mathrm{L} \times \mathrm{G}}(1,44)=9.391$, $p=0.004$; post hoc: forelimbs, $p=0.010, d=0.65)$. No abnormalities in swing time (Fig. $3 C ; F_{\mathrm{G}}(1,44)=0.1209, p>0.05, F_{\mathrm{L}}(1,44)=$ 22.62, $\left.p<0.0001 ; F_{\mathrm{L} \times \mathrm{G}}(1,44)=0.2552, p>0.05\right)$ or total stride time (Fig. $3 D ; F_{\mathrm{G}}(1,44)=0.9166, p>0.05 ; F_{\mathrm{L}}(1,44)=13.24$, $\left.p<0.001 ; F_{\mathrm{L} \times \mathrm{G}}(1,44)=0.7566, p>0.05\right)$ were discovered, suggesting that the opposing effects of propulsion and brake time canceled each other out. Stride length was normal, which was surprising given the published Zeno Walkway data in humans (Grieco et al., 2018), but lends to the hypothesis that Ube $3 a^{\text {mat-/pat+ }}$ have limb weakness since more time 
A

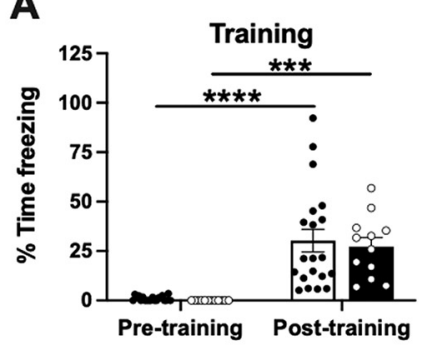

D

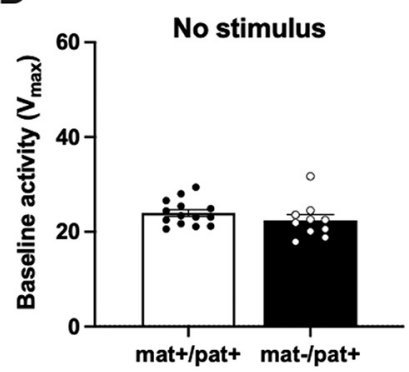

G

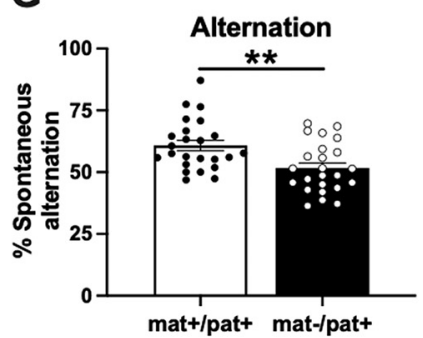

B

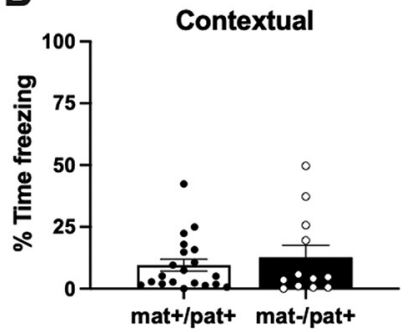

E

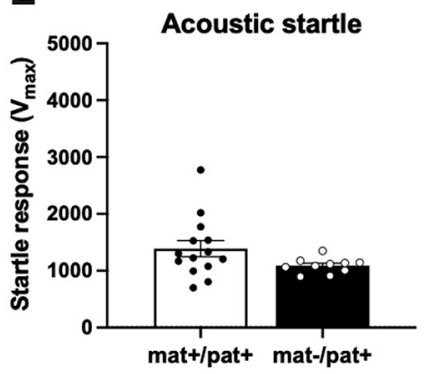

H

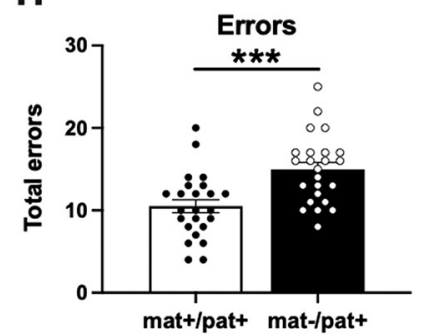

C

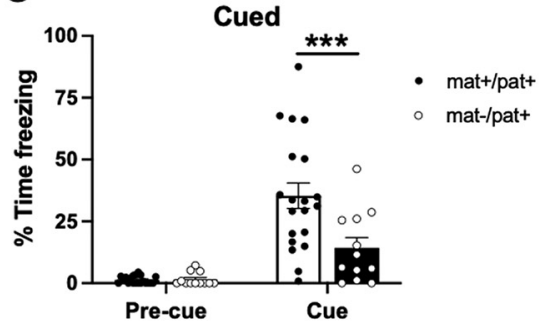

F

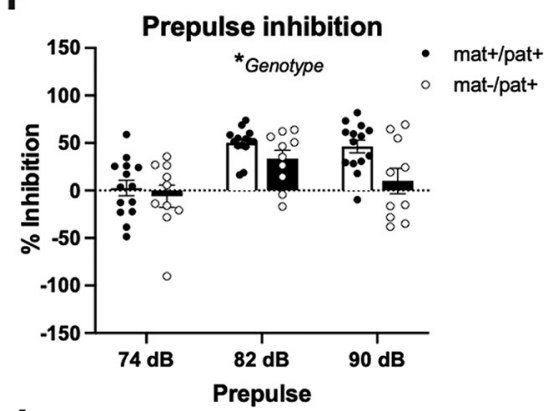

I

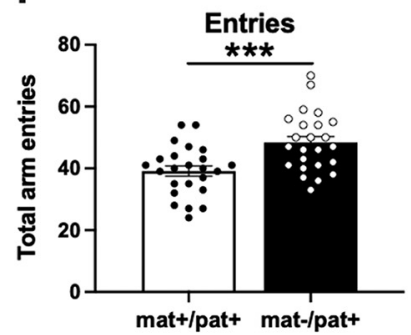

Figure 4. Impaired learning and memory in Ube3a $a^{\text {mat }-/ \text { pat }+}$ rats. $A$, During fear conditioning training, juvenile Ube3a $a^{\text {mat-/pat }+}$ (mat-/pat $+; n=12$ ) and WT littermate controls

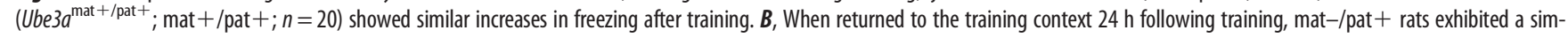
ilar level of freezing to WTs. C, When introduced to a novel context $48 \mathrm{~h}$ after training, no difference in freezing pre-cue was found, but mat-/pat+ rats froze for less than half the time of WTs during presentation of the auditory cue. A separate sensorimotor test confirmed intact auditory sensitivity: $\boldsymbol{D}$, Baseline activity within the testing apparatus was comparable between mat-/pat $+(n=10)$ and mat + pat + rats $(n=14)$. $\boldsymbol{E}$, There was no effect of genotype on the startle response to a 120 decibel (dB) startle stimulus. $\boldsymbol{F}$, Prepulse inhibition of the startle response was generally reduced in adult mat-/pat + rats. G, Spontaneous arm alternation during Y-maze exploration was significantly reduced in adult mat-/pat + rats $(n=24)$ compared with WT littermates $(n=24)$. $\boldsymbol{H}$, Mat-/pat + rats made $40 \%$ more errors and $(\boldsymbol{I})$ made more entries into the maze arms. Error bars indicate mean \pm SEM. $\boldsymbol{A}, \boldsymbol{C}$, ${ }^{* * * *} p<0.0001$, ${ }^{* * *} p<0.001$, repeated-measures ANOVA, Holm-Sidak's post hoc. $\boldsymbol{F},{ }^{*} p<0.05$, repeated-measures ANOVA main effect. $\mathbf{G}-\boldsymbol{I}$, ${ }^{* * *} p<0.001$, ${ }^{* *} p<0.01$, Student's $t$ test.

was required to produce force for an equal length step (Fig. $3 F ; F_{\mathrm{G}}(1,44)=0.9460, p>0.05 ; F_{\mathrm{L}}(1,44)=12.70, p<0.001$; $\left.F_{\mathrm{L} \times \mathrm{G}}(1,44)=0.7719, p>0.05\right)$. Forelimb stance width was reduced (Fig. $3 G ; F_{\mathrm{G}}(1,44)=1.605, p>0.05 ; F_{\mathrm{L}}(1,44)=$ 939.0, $p<0.0001 ; F_{\mathrm{L} \times \mathrm{G}}(1,44)=12.46$; post hoc: forelimbs, $p=0.022, d=0.69$ ) while an elevated forelimb paw angle indicated greater degree of external rotation and splaying (Fig. $3 H ; F_{\mathrm{G}}(1,44)=5.957, p=0.019 ; F_{\mathrm{L}}(1,44)=3.726$, $p>0.05 ; F_{\mathrm{L} \times \mathrm{G}}(1,44)=3.497, p>0.05$; post hoc: forelimbs, $p=0.006, d=0.86$ ), which has been associated with ataxia, spinal cord injury, and demyelinating disease (Powell et al., 1999). The observed effects were not attributable to differences in body length (data not shown; $U=244.5, p>0.05$ ) or body width (data not shown; $t_{(44)}=0.2719, p>0.05$ ); and despite abnormalities in some temporal and postural components of gait, the coordination metric of gait symmetry was unaltered (Fig. $3 I ; t_{(44)}=1.023, p>0.05$ ).

\section{Impaired learning and memory in Ube $3 a^{\text {mat-/pat }+}$ rats}

Learning and memory impairments, which are characteristic of AS, may hinder the ability of $U b e 3 a^{\text {mat }-/ p a t+}$ rats to learn via developmental experience how to appropriately engage in social interactions. We therefore probed for a juvenile learning and memory deficit using a fear conditioning assay previously used to detect a deficit in adulthood (Dodge et al., 2020). Following successful fear conditioning (Fig. $4 A ; F_{\text {Phase }(\mathrm{P})}(1,30)=48.47$, $p<0.0001 ; F_{\text {Genotype(G) }}(1,30)=0.2203, p>0.05 ; F_{\mathrm{P} \times \mathrm{G}}(1,30)=$ $0.0613, p>0.05$; post hoc: mat $+/$ pat,$+ p<0.0001$; mat-/pat + , $p<0.001$ ), juvenile Ube $3 a^{\text {mat-pat }+}$ displayed normal levels of freezing in response to the training context (Fig. $4 B ; U=117.5$, $p>0.05$ ) but a robust deficit in cued fear memory $48 \mathrm{~h}$ after training (Fig. $4 C ; F_{\mathrm{G}}(1,30)=7.395, p=0.011 ; F_{\mathrm{P}}(1,30)=42.36$, $p<0.0001 ; F_{\mathrm{P} \times \mathrm{G}}(1,30)=8.699, p=0.006 ;$ post hoc: pre-cue, $p>0.05$; cue, $p<0.001, d=1.10$ ). We assessed the potentially confounding variable of impaired sensorimotor processing by measuring the startle response to an intense acoustic stimulus and quantifying the reduction in startle response following prepulses of varying intensities. Both baseline activity (Fig. 4D; $\left.t_{(22)}=1.735, p>0.05\right)$ and the acoustic startle response of Ube $3 a^{\text {mat- } / \text { pat }+}$ rats were normal (Fig. $4 E ; t_{(22)}=1.157, p>0.05$ ), indicating intact hearing abilities. While there was a significant main effect of genotype on prepulse inhibition, indicative of a sensorimotor gating deficit $\left(F_{\text {Genotype(G) }}(1,22)=4.740, p=\right.$ $0.041, d=0.88 ; \quad F_{\text {Prepulse(P) }}(1.898,41.75)=20.64, p<0.0001$; 
A

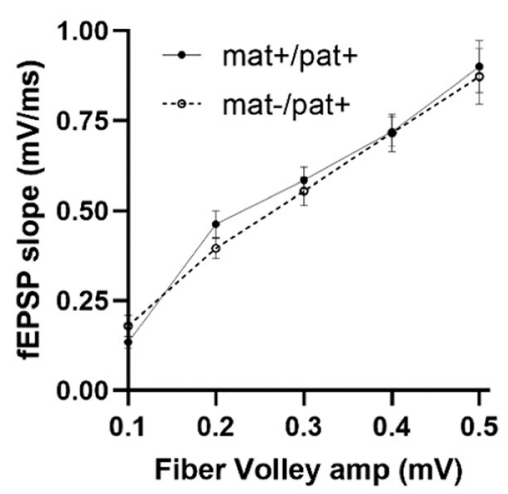

C

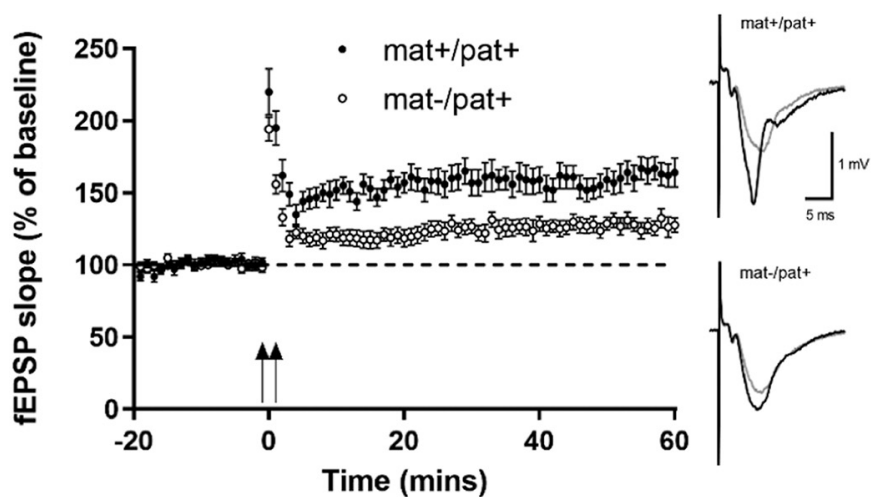

B

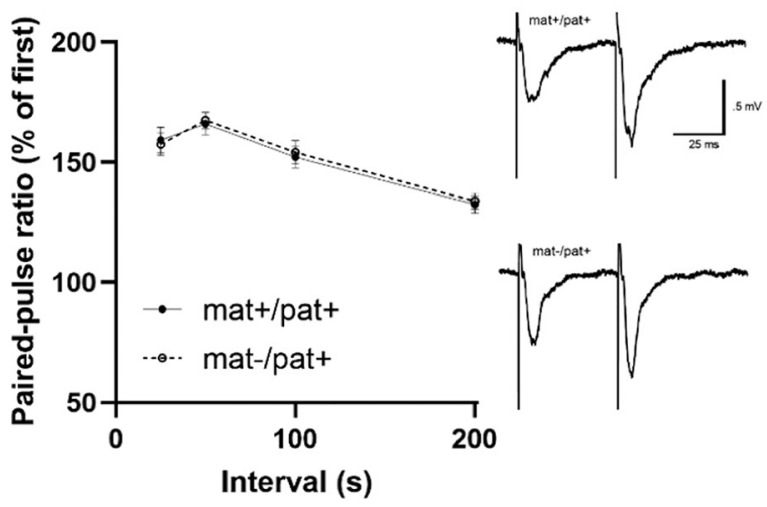

D

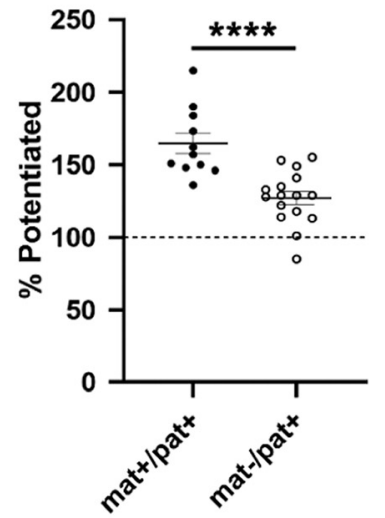

Figure 5. Reduced hippocampal LTP in Ube3amat-/pat+ rats. $\boldsymbol{A}$, Normal basal synaptic transmission as measured by presynaptic fiber volley amplitudes and postsynaptic fEPSP slopes for responses elicited by different intensities of SC fiber stimulation in Ube3at $a^{\text {mat-/pat }+}$ (mat-/pat $\left.+; n=16\right)$ and WT littermate $\left(\right.$ Ube $3 a^{\text {mat }+/ \text { pat }+} ;$ mat $+/$ pat $\left.+; n=11\right)$ hippocampal slices. $\boldsymbol{B}$, Paired-pulse facilitation was unchanged at mat-/pat + SC-CA1 synapses compared with mat $+/$ pat $+(n=15$ mat $+/$ pat + and $n=20$ mat-/pat + slices $)$. Right, Traces represent fEPSPs evoked by stimulation pulses delivered with a 50 ms interpulse interval. Calibration: $0.5 \mathrm{mV}, 25 \mathrm{~ms}$. C, HFS-induced LTP in mat $+/$ pat $+(n=11)$ was significantly greater compared with mat-/pat $+(n=16)$. Right, Traces represent superimposed fEPSPs recorded during baseline and $60 \mathrm{~min}$ after HFS. Calibration: $1 \mathrm{mV}, 5 \mathrm{~ms}$. $\boldsymbol{D}$, Summary graph of average percentage potentiation relative to baseline demonstrating that mat $+/$ pat + exhibited significantly enhanced SC-CA1 LTP at 60 min after HFS (delivered at time $=0$ ), fEPSPs were potentiated to $160 \pm 7 \%$ of baseline in mat $+/$ pat $+(n=11)$ and were $127 \pm 5 \%$ of baseline in mat-/pat + slices $(n=16)$. Data were collected from 2 rats per genotype. ${ }^{* * * *} p<0.0001$, Student's $t$ test.

$\left.F_{\mathrm{P} \times \mathrm{G}}(2,44)=2.127, p=0.1312\right)$, post hoc testing revealed no significant difference between groups at any individual prepulse level (Fig. 4F; $74 \mathrm{~dB}, p>0.05, d=0.25 ; 82 \mathrm{~dB}, p>0.05, d=0.73$; $90 \mathrm{~dB}, p>0.05, d=1.05)$.

As an additional assessment of cognitive functioning, we quantified spontaneous alternation during exploration of a Ymaze and found that $U b e 3 a^{\text {mat-/pat+ }}$ rats displayed reduced spontaneous alternation compared with WTs (Fig. $4 G ; t_{(46)}=$ $3.115, p<0.01, d=0.90)$. Ube $3 a^{\text {mat-/pat }+}$ rats made $40 \%$ more errors (Fig. $4 H ; t_{(46)}=3.827, p<0.001, d=1.10$ ) and more arm entries (Fig. $4 I$; $t_{(46)}=3.620, p<0.001, d=1.04$ ) despite no difference in the total distance moved (data not shown; Student's $t$ test: $\left.t_{(46)}=1.721, p>0.05\right)$. Together, these metrics indicate additional cognitive deficits in the Ube $3 a^{\text {mat-/pat+ }}$ rats that were not confounded by a locomotor deficiency.

Reduced hippocampal LTP in Ube $3 a^{\text {mat-/pat+ }}$ rats

To elucidate the neurobiology underpinning the learning and memory deficits of Ube $3 a^{\text {mat-/pat }+}$ rats, we quantified LTP. Previous studies in mouse models of AS have shown that LTP, a major cellular mechanism underlying learning and memory (Collingridge and Isaac, 2003), is impaired (Jiang et al., 1998; van
Woerden et al., 2007; Daily et al., 2011). Here, we examined hippocampal LTP in adult Ube $3 a^{\text {mat-/pat }+}$ rats compared with WT littermate controls. We found hippocampal-dependent contextual fear memory intact at the juvenile age, but a previous report detected a clear deficit in adults (Dodge et al., 2020), therefore we measured hippocampal LTP in adulthood. Basal synaptic strength (Fig. $5 A ; F_{\text {Genotype }(\mathrm{G})}(1,92)=0.2013, p>0.05 ; F_{\text {Amplitude(A) }}(5,111)=$ 94.04, $\left.p<0.0001 ; F_{\mathrm{G} \times \mathrm{A}}(5,92)=0.4107, p>0.05\right)$ and paired-pulse ratio (Fig. $5 B ; F_{\mathrm{G}}(1,56)=0.065, p>0.05 ; F_{\text {Interval(I) }}(3,76)=20.96$, $\left.p<0.0001 ; F_{\mathrm{G} \times \mathrm{I}}(3,56)=0.0758, p>0.05\right)$ were unaltered in Ube $3 a^{\text {mat-/pat }+}$ rats, suggesting no change in baseline excitatory transmission. However, consistent with the mouse models of AS (Jiang et al., 1998; van Woerden et al., 2007; Daily et al., 2011), we found that the magnitude of LTP was reduced in Ube $3 a^{\text {mat-/pat }+}$ rats (Fig. $5 C, D ; t_{(25)}=4.641, p<0.0001, d=1.78$ ), suggesting a putative mechanism underlying impairment of learning and memory (Zucker, 1989; Jiang et al., 1998; Zucker and Regehr, 2002; van Woerden et al., 2007; Daily et al., 2011).

\section{Neuroanatomical pathology in $U b e 3 a^{\text {mat-/pat+ }}$ rats revealed by high-resolution MRI}

MRI revealed striking differences at 6.5 months of age in total brain volume, which was decreased by $6.0 \%$ in Ube $3 a^{\text {mat-/pat }+}$ rats 

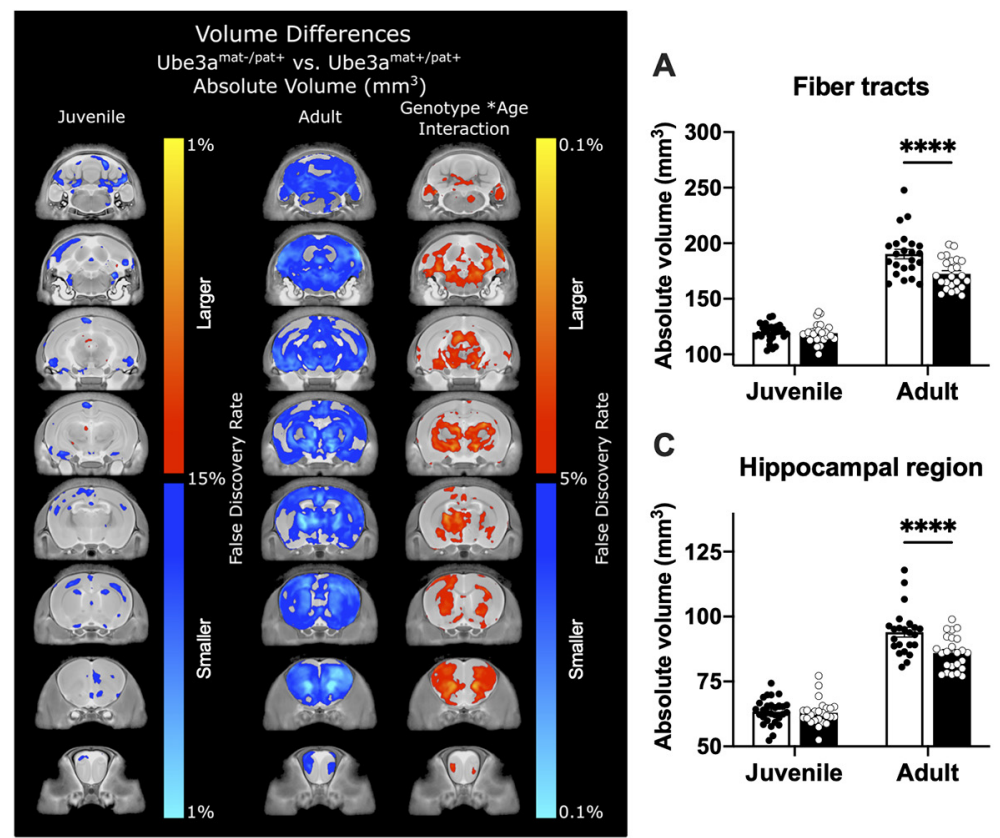

B

Hypothalamus
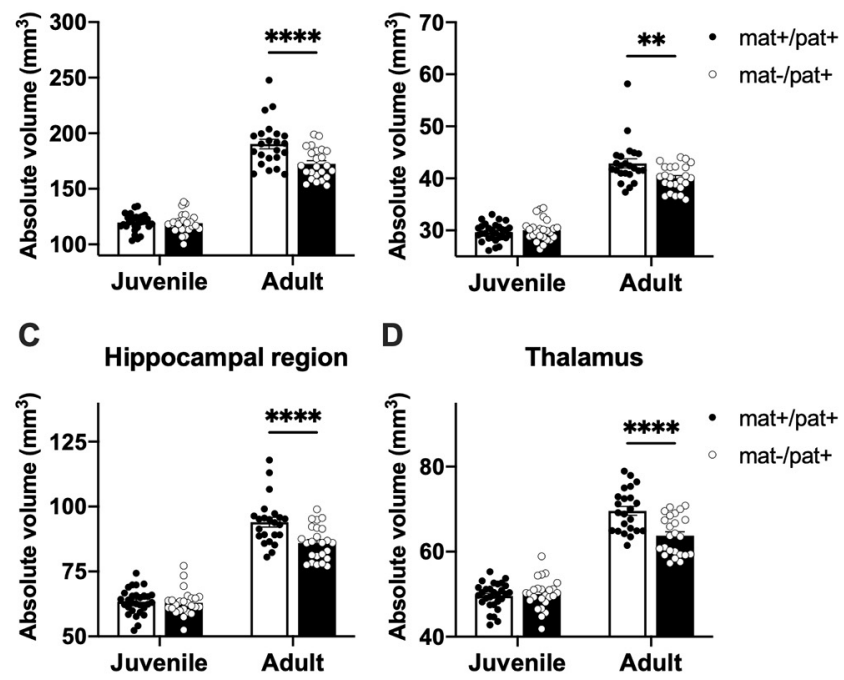

D

Thalamus

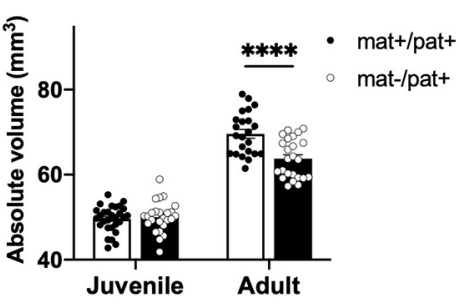

Figure 6. Neuroanatomical pathology in Ube $3 a^{\text {mat-/pat }+}$ rats revealed by high-resolution MRI. Left, Slice series comparing absolute volume $\left(\mathrm{mm}^{3}\right)$ of juvenile and adult populations of Ube $3 a^{\text {mat }-/ \text { pat }+}($ mat $-/$ pat +$)$ rats and WT littermates $\left(U b e 3 a^{\text {mat }+/ \text { pat }+}\right.$; mat $+/$ pat + ). Red to yellow represents increased volume compared with WT. Dark blue to light blue represents decreased volume. Leftmost column represents data on juvenile mat-/pat + rats from Berg et al. (2020c). Middle column represents the same slices on the adult dataset presented here. Most notably, total brain volume was $6.0 \%$ smaller in mat-/pat + rats compared with WT. Additionally, the third column represents the genotype $\times$ age interaction highlighting several R0ls (right), four of which are shown as follows: $\boldsymbol{A}$, fiber tracts; $\boldsymbol{B}$, the hypothalamus; $\boldsymbol{C}$, the hippocampal region; and $\boldsymbol{D}$, the thalamus. Full details of regional findings for adult animals and the interaction effect are described in Extended Data Figures 6-1 and 6-2. Group sizes: juvenile mat $+/$ pat, $n=29$; juvenile mat- $/$ pat,$+ n=25$; adult mat $+/$ pat,$+ n=23$; adult mat $-/$ pat + , $n=24$. Error bars indicate mean \pm SEM. ${ }^{* * *} p<0.0001,{ }^{* *} p<0.01$, two-way ANOVA, Holm-Sidak's post hoc.

( $q=0.04$; Fig. 6; Extended Data Fig. 6-1). The overall brain volume difference was driven by decreases in the hippocampal region $(-6.3 \%, q=0.04)$, brainstem $(-5.6 \%, q=0.04)$, thalamus $(-7.7 \%$, $q=0.01)$, cerebellum $(-9.0 \%, q=0.02)$, and deep cerebellar nuclei $(-12.3 \%, q=0.0001)$. Additional differences were found throughout the white matter fiber tracts $(-7.6 \%, q=0.02)$, including but not limited to the cerebral peduncle $(-7.6 \%, q=0.02)$, internal capsule $(-8.4 \%, q=0.02)$, and arbor vita of the cerebellum $(-11.7 \%$, $q=0.0004)$. Moreover, trends were seen in other large white matter structures, including the corpus callosum $(-6.7 \%, q=0.06)$ and fornix system $(-6.0 \%, q=0.09)$. A complete list of the regional structural differences in both absolute $\left(\mathrm{mm}^{3}\right)$ and relative (\% total brain) volume is provided in Extended Data Fig. 6-1.

As we had previously examined Ube $3 a^{\text {mat-/pat+ }}$ rats at a juvenile age (PND 21) (Berg et al., 2020c), we felt an age $\times$ genotype comparison was warranted. Figure 6 highlights these changes in eight coronal slices, separately from both the previous work on juvenile rats and from the current data on adults. A combined dataset using both the juvenile and adult data were then used to examine a genotype $\times$ age interaction model, which revealed several regions to diverge with age and genotype: total brain volume $(q=0.048)$, caudoputamen $(q=0.03)$, white matter fiber tracts $\left(q=0.03\right.$; Fig. $6 A ; F_{\mathrm{Age}(\mathrm{A})}(1,97)=546.5, p<0.0001$; $F_{\text {Genotype(G) }}(1,97)=11.87, p<0.001 ; F_{\mathrm{A} \times \mathrm{G}}(1,97)=10.68$, $p=0.002$; post hoc: juvenile, $p>0.05$; adult, $p<0.0001, d=1.02$ ), hypothalamus $\left(q=0.046\right.$; Fig. $6 B ; F_{\mathrm{A}}(1,97)=460.2, p<0.0001$; $F_{\mathrm{G}}(1,97)=5.081, p=0.026 ; F_{\mathrm{A} \times \mathrm{G}}(1,97)=8.760, p=0.004 ;$ post hoc: juvenile, $p>0.05$; adult, $p=0.001, d=0.82)$, hippocampal region $\left(q=0.046\right.$; Fig. $6 C ; F_{\mathrm{A}}(1,97)=434.4, p<0.0001$; $F_{\mathrm{G}}(1,97)=10.89, p=0.001 ; F_{\mathrm{A} \times \mathrm{G}}(1,97)=8.760, p=0.004 ;$ post hoc: juvenile, $p>0.05$; adult, $p<0.0001, d=1.02)$, and thalamus $\left(q=0.02\right.$; Fig. $6 D ; F_{\mathrm{A}}(1,97)=430.2, p<0.0001 ; F_{\mathrm{G}}(1,97)=11.14$, $p=0.001 ; F_{\mathrm{A} \times \mathrm{G}}(1,97)=14.96, p<0.001 ;$ post hoc: juvenile, $p>0.05$; adult, $p<0.0001, d=1.22$ ). A full list of the regional genotype $\times$ age interactions is located in Extended Data Fig. 6-2. Voxelwise changes were also found throughout the brain of adult Ube $3 a^{\text {mat-/pat }+}$ rats compared with the juvenile age. The changes in the adults were substantially larger, signaling a more severe neuroanatomical phenotype with age (Fig. 6).

\section{Discussion}

Indispensable to therapeutic development are in vivo studies using preclinical model systems. While mice have prevailed as the animal model of AS in recent decades (Jiang et al., 1998), the Ube $3 a^{\text {mat-/pat+ }}$ rat offers a unique and suitable system for investigating certain complexities of the human AS phenotype, particularly social communication and affect (Brudzynski, 2013; Wöhr and Schwarting, 2013; Burke et al., 2017; Homberg et al., 2017; Braun et al., 2018; Fernández et al., 2018; Burgdorf et al., 2020; Netser et al., 2020). Our discovery of excessive laughter-like $50 \mathrm{kHz}$ USV is the first report of this affective outcome measure in a model of AS, mirroring the affected population. Moreover, reduced social play, atypical gait, impaired cognition, and anatomic and cellular physiology anomalies were easily detected in this model.

We leveraged our model species to discover that Ube $3 a^{\text {mat-/pat+ }}$ rats produced an overabundance of $50 \mathrm{kHz}$ vocalizations, which reflect a positive affective state and have been referred to as rat laughter (Panksepp and Burgdorf, 2000; Panksepp, 2005; Rygula et al., 2012), as well as a trend of elevated laughter-like $50 \mathrm{kHz}$ USV without provocation. Excessive $50 \mathrm{kHz}$ USV, suggestive of enhanced "wanting" and "liking" the interaction (Berridge, 2009; Berridge and Aldridge, 2009; Berridge et al., 2009; Okabe et al., 
2021), closely aligns with the AS profile of a happy disposition and easily provoked laughter. To our knowledge, this is the first report of this method being used in a genetic rat model of a neurological disorder.

Exaggerated $50 \mathrm{kHz}$ calling could suggest enhanced effort to elicit social interaction or may be unrelated to the social component of heterospecific play, potentially a neurobiological consequence of a disinhibited vocal production pathway. AS is typified by laughter that is easily provoked regardless of stimuli valence. The phenotype may also reflect enhanced sensitivity to tactile stimulation. Deriving greater reward from physical interactions could explain typical levels of social investigation in the reciprocal interaction test but reduced social approach in the previously reported three-chambered and USV playback assays, as well as the disinhibition of social interactions in the clinical population. One limitation of our USV analysis was the lack of acoustic feature quantification for the calls evoked by heterospecific play. We did, however, subsequently perform this analysis for all other USV assays and found no genotype effect on call features.

Juvenile social play is a critical way that rats develop social competence and learn how to appropriately engage and communicate with others, analogous to play in young children (Hofer and Shair, 1978; Panksepp and Beatty, 1980; Panksepp, 1981; Brudzynski, 2009, 2013; Argue and McCarthy, 2015). Ube3a $a^{\text {mat-/pat+ }}$ rats were interested in a novel partner but did not engage in rough-and-tumble play behaviors characteristic of the species, albeit specific to sex and strain. Our finding of no sex difference in rough-and-tumble play aligns with previous reports, which also used pretest social isolation to motivate the subjects to play (Veenema et al., 2013; Bredewold et al., 2014, 2015; Reppucci et al., 2018; Kisko et al., 2020). In contrast with studies on mouse models of AS using the three-chambered social approach task (Jamal et al., 2017; Kumar et al., 2019; Dutta and Crawley, 2020; Perrino et al., 2021), which have reported contradictory social deficits and "hypersociability," the rat model displayed a typical level of social investigation.

Movement disorders (Wheeler et al., 2017) are a hallmark feature of AS, with gait ataxia being one of the most common issues. While the deficits of $U b e 3 a^{\text {mat-pat+ }}$ rats were not obvious to the eye, subtle aberrations in stance and paw placement, paired with abnormal braking and propelling, reflect impaired motor coordination. All of this evidence suggests that altered postures affect motor dynamics, which results in the gait patterns exhibited by AS individuals and Ube $3 a^{\text {mat-/pat+ }}$ rats. The limb weakness indicated by our gait analysis aligns with the reduced rearing previously observed (Berg et al., 2020c).

We discovered and report for the first time, to our knowledge, LTP deficits in this rat model (Jiang et al., 1998; Weeber et al., 2003; van Woerden et al., 2007; Filonova et al., 2014; Ciarlone et al., 2016), which provides a putative cellular signaling mechanism underlying the learning and memory impairments reported herein and previously (Berg et al., 2020c; Dodge et al., 2020). Juvenile Ube $3 a^{\text {mat-/pat }+}$ rats exhibited deficits in cued fear memory $48 \mathrm{~h}$ after training, which extends the previous finding by Dodge et al. (2020) of deficient contextual and cued fear conditioning in adults $72 \mathrm{~h}$ after training. We ruled out impaired sensorimotor abilities as a confounding variable since the acoustic startle response was unaffected.

Pronounced deficits in adulthood are supported by neuroimaging. Previously, we discovered a variety of trending volumetric abnormalities at PND 21 (Berg et al., 2020c); however, these new data show more substantial reductions in adults throughout the brain, highlighting a more severe neuroanatomical phenotype with age. Reduced total brain volume may indicate a loss of cellular volume or dendritic complexity over time, and the drastic volume loss in fiber tracts could indicate a loss in axonal numbers, axonal volume, or myelination. In a mouse model of AS, white matter loss was found to play a large role in the overall microcephaly observed (Judson et al., 2017), with an $11 \%$ loss in the corpus callosum making it the most affected white matter structure. Ube $3 a^{\text {mat-/pat+ }}$ rats showed a trend toward reduced corpus callosum volume $(-6.7 \%)$, but the largest white matter deficits were cerebellar. In alignment with Judson et al.'s (2017) study in mice, the reduced fiber tract volume was also disproportionate to the overall brain volume loss, confirming that white matter development plays the major role in the impaired brain growth in AS. Additionally, the $9 \%$ decrease in cerebellum size was consistent with cortical loss $(-9 \%)$, but there was a disproportionate reduction in arbor vitae and deep cerebellar nuclei volume, indicating that the outputs of the cerebellum are impaired.

GABAergic neuron loss (Judson et al., 2016) and decreased tonic inhibition in cerebellar granule cells (Egawa et al., 2012) underlie the theory of brain dysfunction in AS, and hypotheses addressing the theory of reduced inhibitory tone are being pursued for small-molecule development (Ciarlone et al., 2017). The present results are congruent with loss of inhibitory tone as the overarching mechanism theory of AS. Emission of $50 \mathrm{kHz}$ USVs induced by heterospecific play is associated with dopamine release in the nucleus accumbens/ventral striatum (Hori et al., 2013), as is reception of $50 \mathrm{kHz}$ USV (Willuhn et al., 2014). These calls have been considered as signals of "joy," "euphoria," and "laughter," supported by behavioral pharmacology showing increased $50 \mathrm{kHz}$ USVs resulting from amphetamine administration (Brudzynski, 2013, 2015; Wöhr, 2021) and electrical stimulation of reward-associated areas (Burgdorf et al., 2007). Both vocalizations and gait require fine motor control; thus, striatal and motivational components support aberrant frontal-striatal circuitry. Interestingly, the ventral striatum and "reward" associated substrates of the basal ganglia have inhibitory projections, in line with overall theories of AS regarding inhibitory loss.

While gross and fine movement are complicated multisystem physiological processes, AS individuals show ataxic movements in both upper and lower limbs and aberrant gait, suggesting particular involvement of the cerebellum. This was corroborated here and in earlier work by the large reductions in cerebellar nuclei size, which is consistent with our overarching mechanistic hypothesis since Purkinje cell neurons projecting to the deep cerebellar nuclei modulate excitation via inhibition and Egawa et al. (2012) highlighted decreased cerebellar granule cells in AS. Using conditional Ube3a mouse models to identify the neural substrates of circuit hyperexcitability in AS, Judson et al. (2016) provided compelling evidence that GABAergic, but not glutamatergic, Ube3a loss is responsible for mediating the EEG abnormalities and seizures of AS. Previously, we reproduced and extended the Judson et al. (2016) data (Copping and Silverman, 2021), and our hypothesis is that this mechanism extends to social communication, cognitive phenotypes, and impaired gait outcomes, as each shares components of learning and motivation. The loss of brain volume in regions dense with inhibitory neurons seen herein provides further corroborative evidence that GABAergic tone underlies functional outcomes. Independent corroboration comes from ErbB inhibitors, which have been reported to reverse LTP deficits in AS model mice (Kaphzan et al., 2012). While glutamate receptor expression and function were unaltered in Ube3a mice (Kaphzan et al., 2012; Judson et al., 2016), ErbB signaling was shown to rescue LTP impairments 
in Ube3a mice via an increase in inhibitory synaptic transmission, corroborating our core overarching mechanism of reduced inhibitory tone.

In conclusion, we discovered that $U b e 3 a^{\text {mat-/pat+ }}$ rats exhibited interest in a social partner but expressed an atypically high level of laughter-like vocalizations. Deficits in other AS-relevant domains were also discovered, including gait and cognition, and reduced hippocampal LTP. Future lines of investigation will assess the circuitry and mechanisms underlying the excessive laughter-like USV and social-cognitive anomalies in USV reception, in addition to pursuing other neurobiological endpoints. Overall, our results indicate that the deletion of maternal Ube3a in the rat creates a sophisticated rodent model with high face validity to the human AS phenotype. In the pursuit of effective therapeutics, it is essential to be equipped with a diverse set of behavioral outcome measures and neurologic biomarkers by which to assess efficacy. Together, we demonstrate that the Ube $3 a^{\text {mat-/pat+ }}$ rat offers numerous potential outcome measures that are detectable throughout the lifespan.

\section{References}

Adhikari A, Copping NA, Onaga B, Pride MC, Coulson RL, Yang M, Yasui DH, LaSalle JM, Silverman JL (2019) Cognitive deficits in the Snord116 deletion mouse model for Prader-Willi syndrome. Neurobiol Learn Mem 165:106874.

Adhikari A, Copping NA, Beegle J, Cameron DL, Deng P, O'Geen H, Segal DJ, Fink KD, Silverman JL, Anderson JS (2021) Functional rescue in an Angelman syndrome model following treatment with lentivector transduced hematopoietic stem cells. Hum Mol Genet 30:1067-1083.

Albrecht U, Sutcliffe JS, Cattanach BM, Beechey CV, Armstrong D, Eichele G, Beaudet AL (1997) Imprinted expression of the murine Angelman syndrome gene, Ube3a, in hippocampal and Purkinje neurons. Nat Genet 17:75-78.

Argue KJ, McCarthy MM (2015) Utilization of same- vs. mixed-sex dyads impacts the observation of sex differences in juvenile social play behavior. Curr Neurobiol 6:17-23.

Avants BB, Tustison NJ, Song G, Cook PA, Klein A, Gee JC (2011) A reproducible evaluation of ANTs similarity metric performance in brain image registration. Neuroimage 54:2033-2044.

Bailus BJ, Pyles B, McAlister MM, O'Geen H, Lockwood SH, Adams AN, Nguyen JT, Yu A, Berman RF, Segal DJ (2016) Protein delivery of an artificial transcription factor restores widespread ube3a expression in an Angelman syndrome mouse brain. Mol Ther 24:548-555.

Berg EL, Copping NA, Rivera JK, Pride MC, Careaga M, Bauman MD, Berman RF, Lein PJ, Harony-Nicolas H, Buxbaum JD, Ellegood J, Lerch JP, Wöhr M, Silverman JL (2018) Developmental social communication deficits in the Shank3 rat model of Phelan-McDermid syndrome and autism spectrum disorder. Autism Res 11:587-601.

Berg EL, Ching TM, Bruun DA, Rivera JK, Careaga M, Ellegood J, Lerch JP, Wöhr M, Lein PJ, Silverman JL (2020a) Translational outcomes relevant to neurodevelopmental disorders following early life exposure of rats to chlorpyrifos. J Neurodev Disord 12:40.

Berg EL, Pedersen LR, Pride MC, Petkova SP, Patten KT, Valenzuela AE, Wallis C, Bein KJ, Wexler A, Lein PJ, Silverman JL (2020b) Developmental exposure to near roadway pollution produces behavioral phenotypes relevant to neurodevelopmental disorders in juvenile rats. Transl Psychiatry 10:289.

Berg EL, Pride MC, Petkova SP, Lee RD, Copping NA, Shen Y, Adhikari A, Fenton TA, Pedersen LR, Noakes LS, Nieman BJ, Lerch JP, Harris S, Born HA, Peters MM, Deng P, Cameron DL, Fink KD, Beitnere U, O'Geen H, et al. (2020c) Translational outcomes in a full gene deletion of ubiquitin protein ligase E3A rat model of Angelman syndrome. Transl Psychiatry 10:39.

Berridge KC (2009) 'Liking' and 'wanting' food rewards: brain substrates and roles in eating disorders. Physiol Behav 97:537-550.

Berridge KC, Aldridge JW (2009) Decision utility, incentive salience, and cue-triggered 'wanting.' Oxf Ser Soc Cogn Soc Neurosci 2009:509-533.

Berridge KC, Robinson TE, Aldridge JW (2009) Dissecting components of reward: 'liking', 'wanting', and learning. Curr Opin Pharmacol 9:65-73.
Bird LM (2014) Angelman syndrome: review of clinical and molecular aspects. Appl Clin Genet 7:93-104.

Bishop J, Feintuch A, Bock NA, Nieman B, Dazai J, Davidson L, Henkelman RM (2006) Retrospective gating for mouse cardiac MRI. Magn Reson Med 55:472-477.

Blanchard RJ, Blanchard DC, Agullana R, Weiss SM (1991) Twenty-two kHz alarm cries to presentation of a predator, by laboratory rats living in visible burrow systems. Physiol Behav 50:967-972.

Bock NA, Nieman BJ, Bishop JB, Henkelman RM (2005) In vivo multiplemouse MRI at 7 Tesla. Magn Reson Med 54:1311-1316.

Braun MD, Kisko TM, Vecchia DD, Andreatini R, Schwarting RK, Wöhr M (2018) Sex-specific effects of Cacnalc haploinsufficiency on object recognition, spatial memory, and reversal learning capabilities in rats. Neurobiol Learn Mem 155:543-555.

Bredewold R, Smith CJ, Dumais KM, Veenema AH (2014) Sex-specific modulation of juvenile social play behavior by vasopressin and oxytocin depends on social context. Frontiers in behavioral neuroscience 8:216.

Bredewold R, Schiavo JK, van der Hart M, Verreii M, Veenema AH (2015) Dynamic changes in extracellular release of GABA and glutamate in the lateral septum during social play behavior in juvenile rats: implications for sex-specific regulation of social play behavior. Neuroscience 307:117127.

Brudzynski SM (2009) Communication of adult rats by ultrasonic vocalization: biological, sociobiological, and neuroscience approaches. ILAR J 50:43-50.

Brudzynski SM (2013) Ethotransmission: communication of emotional states through ultrasonic vocalization in rats. Curr Opin Neurobiol 23:310-317.

Brudzynski SM (2015) Pharmacology of ultrasonic vocalizations in adult rats: significance, call classification and neural substrate. Curr Neuropharmacol 13:180-192.

Burgdorf JS, Brudzynski SM, Moskal JR (2020) Using rat ultrasonic vocalization to study the neurobiology of emotion: from basic science to the development of novel therapeutics for affective disorders. Curr Opin Neurobiol 60:192-200.

Burgdorf J, Kroes RA, Moskal JR, Pfaus JG, Brudzynski SM, Panksepp J (2008) Ultrasonic vocalizations of rats (Rattus norvegicus) during mating, play, and aggression: behavioral concomitants, relationship to reward, and self-administration of playback. J Comp Psychol 122:357-367.

Burgdorf J, Panksepp J (2001) Tickling induces reward in adolescent rats. Physiol Behav 72:167-173.

Burgdorf J, Panksepp J, Brudzynski SM, Kroes R, Moskal JR (2005) Breeding for $50 \mathrm{kHz}$ positive affective vocalization in rats. Behav Genet 35:67-72.

Burgdorf J, Wood PL, Kroes RA, Moskal JR, Panksepp J (2007) Neurobiology of $50-\mathrm{kHz}$ ultrasonic vocalizations in rats: Electrode mapping, lesion, and pharmacology studies. Behavioural Brain Research 182:274-283.

Burke CJ, Kisko TM, Swiftwolfe H, Pellis SM, Euston DR (2017) Specific 50 $\mathrm{kHz}$ vocalizations are tightly linked to particular types of behavior in juvenile rats anticipating play. PLoS One 12:e0175841.

Ciarlone SL, Grieco JC, D’Agostino DP, Weeber EJ (2016) Ketone ester supplementation attenuates seizure activity, and improves behavior and hippocampal synaptic plasticity in an Angelman syndrome mouse model. Neurobiol Dis 96:38-46.

Ciarlone SL, Wang X, Rogawski MA, Weeber EJ (2017) Effects of the synthetic neurosteroid ganaxolone on seizure activity and behavioral deficits in an Angelman syndrome mouse model. Neuropharmacology 116:142150 .

Collingridge GL, Isaac JT (2003) Functional roles of protein interactions with AMPA and kainate receptors. Neurosci Res 47:3-15.

Collins DL, Neelin P, Peters TM, Evans AC (1994) Automatic 3D intersubject registration of MR volumetric data in standardized Talairach space. J Comput Assist Tomogr 18:192-205.

Copping NA, Silverman JL (2021) Abnormal electrophysiological phenotypes and sleep deficits in a mouse model of Angelman Syndrome. Mol Autism 12:1-14.

Copping NA, Christian SG, Ritter DJ, Islam MS, Buscher N, Zolkowska D, Pride MC, Berg EL, LaSalle JM, Ellegood J, Lerch JP, Reiter LT, Silverman JL, Dindot SV (2017) Neuronal overexpression of Ube3a isoform 2 causes behavioral impairments and neuroanatomical pathology relevant to 15q11.2-q13.3 duplication syndrome. Hum Mol Genet 26:3995-4010. 
Daily JL, Nash K, Jinwal U, Golde T, Rogers J, Peters MM, Burdine RD, Dickey C, Banko JL, Weeber EJ (2011) Adeno-associated virus-mediated rescue of the cognitive defects in a mouse model for Angelman syndrome. PLoS One 6:e27221.

Dodge A, Peters MM, Greene HE, Dietrick C, Botelho R, Chung D, Willman J, Nenninger AW, Ciarlone S, Kamath SG, Houdek P, Sumova A, Anderson AE, Dindot SV, Berg EL, O'Geen H, Segal DJ, Silverman JL, Weeber EJ, Nash KR (2020) Generation of a novel rat model of Angelman syndrome with a complete Ube3a gene deletion. Autism Res 13:397-409.

Dorr AE, Lerch JP, Spring S, Kabani N, Henkelman RM (2008) High resolution three-dimensional brain atlas using an average magnetic resonance image of 40 adult C57Bl/6J mice. Neuroimage 42:60-69.

Dutta R, Crawley JN (2020) Behavioral evaluation of Angelman syndrome mice at older ages. Neuroscience 445:163-171.

Egawa K, Kitagawa K, Inoue K, Takayama M, Takayama C, Saitoh S, Kishino T, Kitagawa M, Fukuda A (2012) Decreased tonic inhibition in cerebellar granule cells causes motor dysfunction in a mouse model of Angelman syndrome. Sci Transl Med 4:163ra157. ra

Ellenbroek B, Youn J (2016) Rodent models in neuroscience research: is it a rat race? Dis Model Mech 9:1079-1087.

Fendt M, Brosch M, Wernecke KE, Willadsen M, Wöhr M (2018) Predator odour but not TMT induces $22 \mathrm{kHz}$ ultrasonic vocalizations in rats that lead to defensive behaviours in conspecifics upon replay. Sci Rep 8:11041.

Fernández M, Mollinedo-Gajate I, Peñagarikano O (2018) Neural circuits for social cognition: implications for autism. Neuroscience 370:148-162.

Filonova I, Trotter JH, Banko JL, Weeber EJ (2014) Activity-dependent changes in MAPK activation in the Angelman Syndrome mouse model. Learn Mem 21:98-104.

Genovese CR, Lazar NA, Nichols T (2002) Thresholding of statistical maps in functional neuroimaging using the false discovery rate. Neuroimage 15:870-878.

Grieco JC, Gouelle A, Weeber EJ (2018) Identification of spatiotemporal gait parameters and pressure-related characteristics in children with Angelman syndrome: a pilot study. J Appl Res Intellect Disabil 31:12191224.

Hammerschmidt K, Reisinger E, Westekemper K, Ehrenreich L, Strenzke N, Fischer J (2012) Mice do not require auditory input for the normal development of their ultrasonic vocalizations. BMC Neurosci 13:40.

Harony-Nicolas H, Kay M, du Hoffmann J, Klein ME, Bozdagi-Gunal O, Riad M, Daskalakis NP, Sonar S, Castillo PE, Hof PR, Shapiro ML, Baxter MG, Wagner S, Buxbaum JD (2017) Oxytocin improves behavioral and electrophysiological deficits in a novel Shank3-deficient rat. Elife 6: e18904.

Hofer MA, Shair H (1978) Ultrasonic vocalization during social interaction and isolation in 2-weeek-old rats. Dev Psychobiol 11:495-504.

Hofer MA, Shair HN, Brunelli SA (2002) Ultrasonic vocalizations in rat and mouse pups. Curr Protoc Neurosci Chapter 8:Unit 8.14.

Homberg JR, Wöhr M, Alenina N (2017) Comeback of the rat in biomedical research. ACS Chem Neurosci 8:900-903.

Hori M, Shimoju R, Tokunaga R, Ohkubo M, Miyabe S, Ohnishi J, Murakami K, Kurosawa M (2013) Tickling increases dopamine release in the nucleus accumbens and $50 \mathrm{kHz}$ ultrasonic vocalizations in adolescent rats. NeuroReport 24:241-245.

Huang HS, Burns AJ, Nonneman RJ, Baker LK, Riddick NV, Nikolova VD, Riday TT, Yashiro K, Philpot BD, Moy SS (2013) Behavioral deficits in an Angelman syndrome model: effects of genetic background and age. Behav Brain Res 243:79-90.

Ishiyama S, Brecht M (2016) Neural correlates of ticklishness in the rat somatosensory cortex. Science 354:757-760.

Jamal I, Kumar V, Vatsa N, Shekhar S, Singh BK, Sharma A, Jana NR (2017) Rescue of altered HDAC activity recovers behavioural abnormalities in a mouse model of Angelman syndrome. Neurobiol Dis 105:99-108.

Jiang YH, Armstrong D, Albrecht U, Atkins CM, Noebels JL, Eichele G, Sweatt JD, Beaudet AL (1998) Mutation of the Angelman ubiquitin ligase in mice causes increased cytoplasmic p53 and deficits of contextual learning and long-term potentiation. Neuron 21:799-811.

Jiang YH, Pan Y, Zhu L, Landa L, Yoo J, Spencer C, Lorenzo I, Brilliant M, Noebels J, Beaudet AL (2010) Altered ultrasonic vocalization and impaired learning and memory in Angelman syndrome mouse model with a large maternal deletion from Ube3a to Gabrb3. PLoS One 5: e12278.
Judson MC, Burette AC, Thaxton CL, Pribisko AL, Shen MD, Rumple AM, Del Cid WA, Paniagua B, Styner M, Weinberg RJ, Philpot BD (2017) Decreased axon caliber underlies loss of fiber tract integrity, disproportional reductions in white matter volume, and microcephaly in Angelman syndrome model mice. J Neurosci 37:7347-7361.

Judson MC, Wallace ML, Sidorov MS, Burette AC, Gu B, van Woerden GM, King IF, Han JE, Zylka MJ, Elgersma Y, Weinberg RJ, Philpot BD (2016) GABAergic neuron-specific loss of Ube3a causes Angelman syndromelike EEG abnormalities and enhances seizure susceptibility. Neuron 90:56-69.

Kaphzan H, Hernandez P, Jung JI, Cowansage KK, Deinhardt K, Chao MV, Abel T, Klann E (2012) Reversal of impaired hippocampal long-term potentiation and contextual fear memory deficits in Angelman syndrome model mice by ErbB inhibitors. Biol Psychiatry 72:182-190.

Kisko TM, Wöhr M, Pellis VC, Pellis SM (2017) From play to aggression: high-frequency $50 \mathrm{kHz}$ ultrasonic vocalizations as play and appeasement signals in rats. Curr Top Behav Neurosci 30:91-108.

Kisko TM, Braun MD, Michels S, Witt SH, Rietschel M, Culmsee C, Schwarting RK, Wöhr M (2018) Cacnalc haploinsufficiency leads to prosocial $50 \mathrm{kHz}$ ultrasonic communication deficits in rats. Dis Model Mech 11:dmm034116.

Kisko TM, Braun MD, Michels S, Witt SH, Rietschel M, Culmsee C, Schwarting RK, Wöhr M (2020) Sex-dependent effects of Cacnalc haploinsufficiency on juvenile social play behavior and pro-social $50 \mathrm{kHz}$ ultrasonic communication in rats. Genes Brain Behav 19:e12552.

Kondrakiewicz K, Kostecki M, Szadzińska W, Knapska E (2019) Ecological validity of social interaction tests in rats and mice. Genes Brain Behav 18: e12525.

Kumar V, Joshi T, Vatsa N, Singh BK, Jana NR (2019) Simvastatin restores HDAC1/2 activity and improves behavioral deficits in Angelman syndrome model mouse. Front Mol Neurosci 12:289.

Leach PT, Crawley JN (2018) Touchscreen learning deficits in Ube3a, Ts65Dn and Mecp2 mouse models of neurodevelopmental disorders with intellectual disabilities. Genes Brain Behav 17:e12452.

Lerch JP, Carroll JB, Spring S, Bertram LN, Schwab C, Hayden MR, Henkelman RM (2008a) Automated deformation analysis in the YAC128 Huntington disease mouse model. Neuroimage 39:32-39.

Lerch JP, Pruessner J, Zijdenbos AP, Collins DL, Teipel SJ, Hampel H, Evans AC (2008b) Automated cortical thickness measurements from MRI can accurately separate Alzheimer's patients from normal elderly controls. Neurobiol Aging 29:23-30.

Lerch JP, Carroll JB, Dorr A, Spring S, Evans AC, Hayden MR, Sled JG, Henkelman RM (2008c) Cortical thickness measured from MRI in the YAC128 mouse model of Huntington's disease. Neuroimage 41:243-251.

Lerch JP, Sled JG, Henkelman RM (2011) MRI phenotyping of genetically altered mice. Methods Mol Biol 711:349-361.

Meaney MJ, Stewart J (1981) Neonatal-androgens influence the social play of prepubescent rats. Horm Behav 15:197-213.

Meng L, Ward AJ, Chun S, Bennett CF, Beaudet AL, Rigo F (2015) Towards a therapy for Angelman syndrome by targeting a long non-coding RNA. Nature 518:409-412.

Netser S, Meyer A, Magalnik H, Zylbertal A, de la Zerda SH, Briller M, Bizer A, Grinevich V, Wagner S (2020) Distinct dynamics of social motivation drive differential social behavior in laboratory rat and mouse strains. Nat Commun 11:5908.

Nieman BJ, Shyu JY, Rodriguez JJ, Garcia AD, Joyner AL, Turnbull DH (2010) In vivo MRI of neural cell migration dynamics in the mouse brain. Neuroimage 50:456-464.

Nieman BJ, van Eede MC, Spring S, Dazai J, Henkelman RM, Lerch JP (2018) MRI to assess neurological function. Curr Protoc Mouse Biol 8: e44.

Okabe S, Takayanagi Y, Yoshida M, Onaka T (2021) Post-weaning stroking stimuli induce affiliative behavior toward humans and influence brain activity in female rats. Sci Rep 11:3805.

Panksepp J (1981) The ontogeny of play in rats. Dev Psychobiol 14:327-332.

Panksepp J (2005) Psychology. Beyond a joke: from animal laughter to human joy? Science 308:62-63.

Panksepp J, Beatty WW (1980) Social deprivation and play in rats. Behav Neural Biol 30:197-206

Panksepp J, Burgdorf J (2000) $50 \mathrm{kHz}$ chirping (laughter?) in response to conditioned and unconditioned tickle-induced reward in rats: effects of social housing and genetic variables. Behav Brain Res 115:25-38. 
Parker CC, Chen H, Flagel SB, Geurts AM, Richards JB, Robinson TE, Solberg Woods LC, Palmer AA (2014) Rats are the smart choice: rationale for a renewed focus on rats in behavioral genetics. Neuropharmacology 76:250-258.

Pellis SM, Pellis VC (1998) Play fighting of rats in comparative perspective: a schema for neurobehavioral analyses. Neurosci Biobehav Rev 23:87-101.

Pellis SM, Pellis VC (2017) What is play fighting and what is it good for? Learn Behav 45:355-366.

Perrino PA, Chamberlain SJ, Eigsti IM, Fitch RH (2021) Communicationrelated assessments in an Angelman syndrome mouse model. Brain Behav 11:e01937.

Portfors CV (2007) Types and functions of ultrasonic vocalizations in laboratory rats and mice. J Am Assoc Lab Anim Sci 46:28-34.

Portfors CV, Perkel DJ (2014) The role of ultrasonic vocalizations in mouse communication. Curr Opin Neurobiol 28:115-120.

Powell E, Anch AM, Dyche J, Bloom C, Richtert RR (1999) The splay angle: a new measure for assessing neuromuscular dysfunction in rats. Physiol Behav 67:819-821.

Reppucci CJ, Gergely CK, Veenema AH (2018) Activation patterns of vasopressinergic and oxytocinergic brain regions following social play exposure in juvenile male and female rats. Journal of neuroendocrinology 30 : e12582.

Reppucci CJ, Brown LA, Chambers AQ, Veenema AH (2020) Wistar rats and $\mathrm{C} 57 \mathrm{BL} / 6$ mice differ in their motivation to seek social interaction versus food in the Social versus Food Preference Test. Physiol Behav 227:113162.

Rygula R, Pluta H, Popik P (2012) Laughing rats are optimistic. PLoS One 7: e51959.

Sadananda M, Wöhr M, Schwarting RK (2008) Playback of $22 \mathrm{kHz}$ and 50 $\mathrm{kHz}$ ultrasonic vocalizations induces differential $\mathrm{c}$-fos expression in rat brain. Neurosci Lett 435:17-23.

Schwarting RK, Jegan N, Wöhr M (2007) Situational factors, conditions and individual variables which can determine ultrasonic vocalizations in male adult Wistar rats. Behav Brain Res 182:208-222.

Spencer Noakes TL, Henkelman RM, Nieman BJ (2017) Partitioning k-space for cylindrical three-dimensional rapid acquisition with relaxation enhancement imaging in the mouse brain. NMR Biomed 30:11.

Steadman PE, Ellegood J, Szulc KU, Turnbull DH, Joyner AL, Henkelman RM, Lerch JP (2014) Genetic effects on cerebellar structure across mouse models of autism using a magnetic resonance imaging atlas. Autism Res 7:124-137.

van Woerden GM, Harris KD, Hojjati MR, Gustin RM, Qiu S, de Avila Freire R, Jiang YH, Elgersma Y, Weeber EJ (2007) Rescue of neurological deficits in a mouse model for Angelman syndrome by reduction of alphaCaMKII inhibitory phosphorylation. Nat Neurosci 10:280-282.

Veenema AH, Bredewold R, De Vries GJ (2013) Sex-specific modulation of juvenile social play by vasopressin. Psychoneuroendocrinology 38:25542561.

Weeber EJ, Jiang YH, Elgersma Y, Varga AW, Carrasquillo Y, Brown SE, Christian JM, Mirnikjoo B, Silva A, Beaudet AL, Sweatt JD (2003) Derangements of hippocampal calcium/calmodulin-dependent protein kinase II in a mouse model for Angelman mental retardation syndrome. J Neurosci 23:2634-2644.

Wheeler AC, Sacco P, Cabo R (2017) Unmet clinical needs and burden in Angelman syndrome: a review of the literature. Orphanet J Rare Dis 12:164.

Williams C, Franco L (2010) Angelman syndrome at the synapse: meeting report of the Angelman Syndrome Foundation's 2009 Scientific Symposium. J Child Neurol 25:254-261.

Willuhn I, Tose A, Wanat MJ, Hart AS, Hollon NG, Phillips PE, Schwarting RK, Wöhr M (2014) Phasic dopamine release in the nucleus accumbens in response to pro-social $50 \mathrm{kHz}$ ultrasonic vocalizations in rats. J Neurosci 34:10616-10623.

Wöhr M (2021) Measuring mania-like elevated mood through amphetamine-induced $50 \mathrm{kHz}$ ultrasonic vocalizations in rats. $\mathrm{Br} \mathrm{J}$ Pharmacol 2021:1-19.

Wöhr M, Schwarting RK (2007) Ultrasonic communication in rats: can playback of $50 \mathrm{kHz}$ calls induce approach behavior? PLoS One 2:e1365.

Wöhr M, Schwarting RK (2013) Affective communication in rodents: ultrasonic vocalizations as a tool for research on emotion and motivation. Cell Tissue Res 354:81-97.

Wöhr M, Houx B, Schwarting RK, Spruijt B (2008) Effects of experience and context on $50 \mathrm{kHz}$ vocalizations in rats. Physiol Behav 93:766-776.

Wöhr M, Kehl M, Borta A, Schanzer A, Schwarting RK, Hoglinger GU (2009) New insights into the relationship of neurogenesis and affect: tickling induces hippocampal cell proliferation in rats emitting appetitive 50 $\mathrm{kHz}$ ultrasonic vocalizations. Neuroscience 163:1024-1030.

Wöhr M, Seffer D, Schwarting RK (2016) Studying socio-affective communication in rats through playback of ultrasonic vocalizations. Curr Protoc Neurosci 75:8.35.1-8.35.17.

Wolter JM, Mao H, Fragola G, Simon JM, Krantz JL, Bazick HO, Oztemiz B, Stein JL, Zylka MJ (2020) Cas9 gene therapy for Angelman syndrome traps Ube3a-ATS long non-coding RNA. Nature 587:281-284.

Zucker RS (1989) Short-term synaptic plasticity. Annu Rev Neurosci 12:1331

Zucker RS, Regehr WG (2002) Short-term synaptic plasticity. Annu Rev Physiol 64:355-405. 